\title{
Supporting The Injured Athlete: Coaches' Perspectives On Providing Social Support
}

\author{
Stefanee Opal Maurice \\ West Virginia University, stmaurice@mail.wvu.edu
}

Follow this and additional works at: https://researchrepository.wvu.edu/etd

Part of the Sports Studies Commons

\section{Recommended Citation}

Maurice, Stefanee Opal, "Supporting The Injured Athlete: Coaches' Perspectives On Providing Social Support" (2019). Graduate Theses, Dissertations, and Problem Reports. 4017.

https://researchrepository.wvu.edu/etd/4017

This Dissertation is protected by copyright and/or related rights. It has been brought to you by the The Research Repository @ WVU with permission from the rights-holder(s). You are free to use this Dissertation in any way that is permitted by the copyright and related rights legislation that applies to your use. For other uses you must obtain permission from the rights-holder(s) directly, unless additional rights are indicated by a Creative Commons license in the record and/ or on the work itself. This Dissertation has been accepted for inclusion in WVU Graduate Theses, Dissertations, and Problem Reports collection by an authorized administrator of The Research Repository @ WVU.

For more information, please contact researchrepository@mail.wvu.edu. 


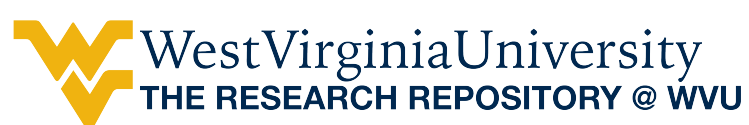

Graduate Theses, Dissertations, and Problem Reports

2019

SUPPORTING THE INJURED ATHLETE:

COACHES' PERSPECTIVES ON PROVIDING SOCIAL SUPPORT

Stefanee Opal Maurice

Follow this and additional works at: https://researchrepository.wvu.edu/etd

Part of the Sports Studies Commons 


\title{
SUPPORTING THE INJURED ATHLETE: COACHES' PERSPECTIVES ON PROVIDING SOCIAL SUPPORT
}

\author{
Stefanee Maurice \\ Dissertation submitted \\ to the College of Physical Activities and Sport Sciences \\ at West Virginia University \\ in partial fulfillment of the requirements for the degree of \\ Doctor of Philosophy in \\ Sport and Exercise Psychology
}

Dana K. Voelker, Ph.D., Chair

Clayton R. Kuklick, Ph.D.

Damien Clement, Ph.D.

Jack C. Watson, II, Ph.D.

Department of Sport Sciences

Morgantown, WV

2019

Keywords: rehabilitation, coach-athlete relationship, sport injury, coaching

Copyright 2019 Stefanee Maurice 


\section{Abstract \\ SUPPORTING THE INJURED ATHLETE: COACHES’ PERSPECTIVES ON PROVIDING SOCIAL SUPPORT}

\section{by Stefanee Maurice}

Injury is an inevitable facet of sport participation, and injured athletes require support from coaches. However, research on injured athletes highlights a lack of support from coaches. Building on the conceptual model proposed by Maurice et al., this study uses the International Sport Coaching Framework (ISCF) to examine ways contextual coaching knowledge is used to support athletes throughout rehabilitation. Previous research has focused on the knowledge types but has neither addressed the integration of the knowledges in a single study nor examined them in an injury context. A generic qualitative approach was used to examine 13 NCAA DI coaches' perception of their role during rehabilitation, their use of ISCF knowledge types, and perceived barriers when supporting injured athletes. Analyzed using deductive coding strategies, coaches reported integrating the knowledge types when supporting their injured athletes. Coaches' perceived roles and barriers were also addressed by the knowledge types. Coaches emphasized their role during the rehabilitation process was to continue communicating with their athletes and know their players well enough to push them to return to play without furthering their athletes' injuries. Barriers perceived by coaches in their efforts to provide support came from rules developed by universities and NCAA limiting ways coaches could offer support. Coaches cited injured athletes as barriers, explaining that athletes are not always honest with coaches about injury severity and afraid to admit they are injured. Results of this study can be used to help coaches identify effective uses of coaching knowledge to improve injured athletes' rehabilitation experiences. 


\section{ACKNOWLEDGMENTS}

The completion of this dissertation would not have been possible without the numerous sources of social support I found. First, I would like to thank my chair, Dr. Dana Voelker, for her continuous support and guidance throughout this process. Dr. Voelker has been dedicated to helping me get to the finish line and I am so appreciative of all she has done to get me here. I would also like to thank my committee members Drs. Kuklick, Watson, and Clement for their time and expertise that has helped me get to this point. The staff within the college have been instrumental sources of both informational and emotional support throughout the entire process. I would like to express my gratitude to faculty in the counseling department and in the department of sport sciences, and my supervisors at the Carruth Center for supporting over the years and through this project. I would also like to acknowledge the funding support of the NCAA Graduate Student Research Grant and the coaches who took part in this study.

To my students and colleagues at Cal Poly, thank you for all you have done to support me and my research. I am so lucky to have found such a supportive home in academia.

Through the many ups and downs of completing this project, I was fortunate to have the unwavering support of friends like Megan Byrd, Carra Johnson, Meghan Halbrook, Tammy Sheehy, Ashley Samson, and so many others. I would also like to thank my family for their continuous support of my educational pursuits and always believing in me. 
Table of Contents

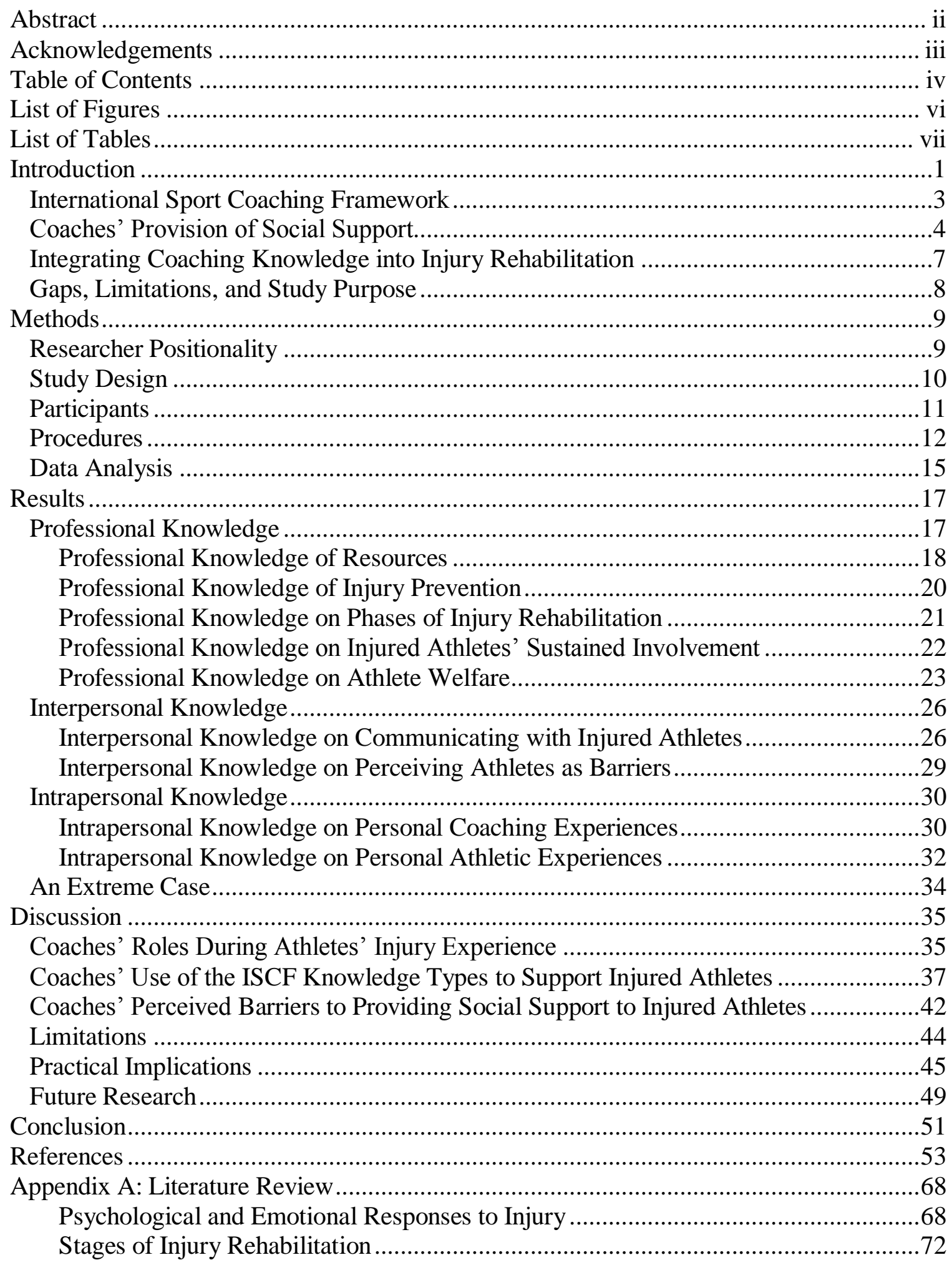




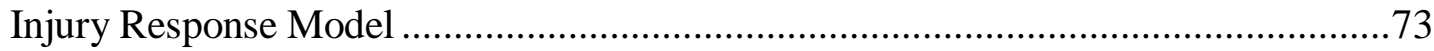

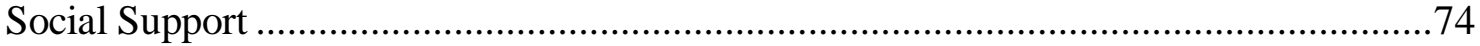

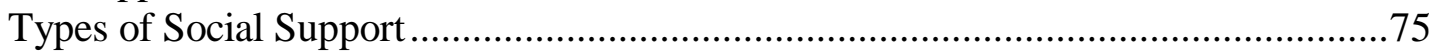

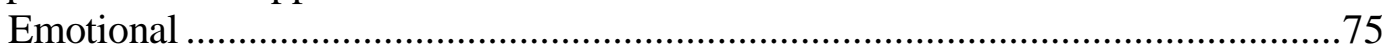

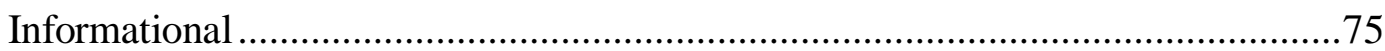

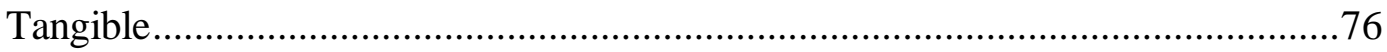

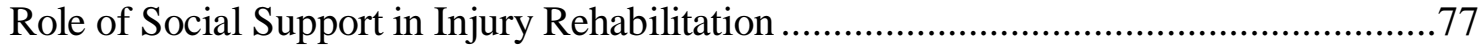

Coaches as a Source of Social Support ..................................................................... 79

International Sport Coaching Framework .......................................................... 83

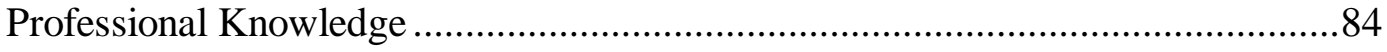

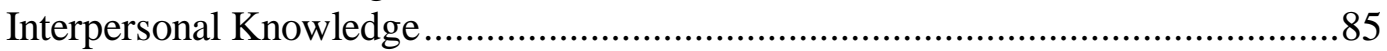

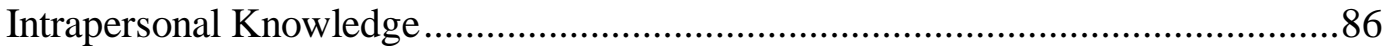

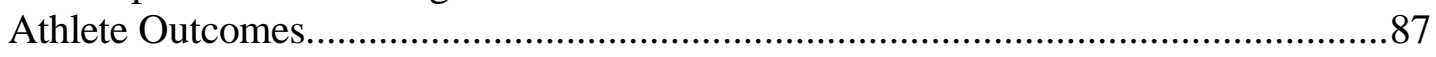

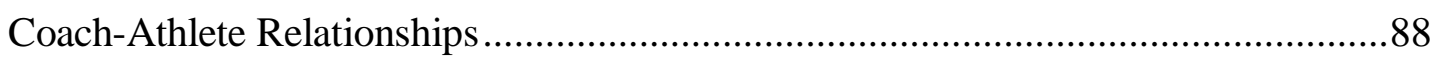

Appendix B: Assumptions, Key Terms, and Limitations ............................................ 90

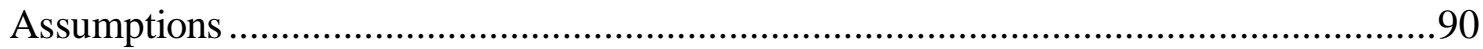

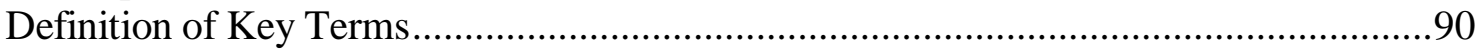

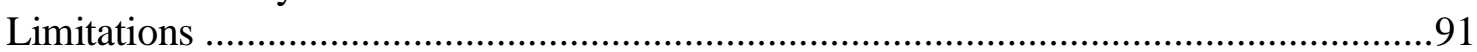

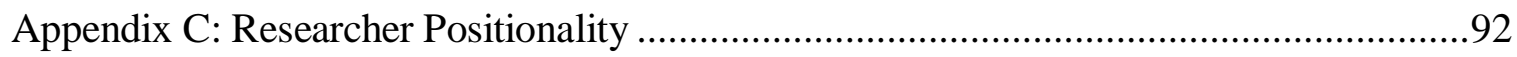

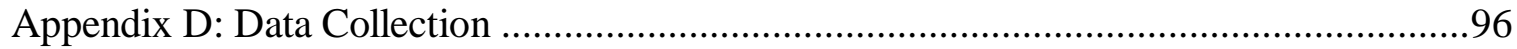

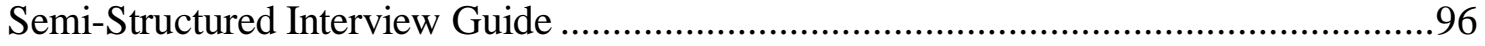

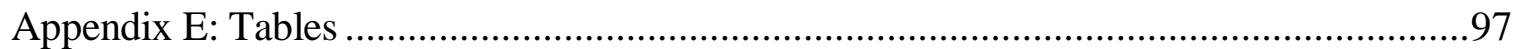

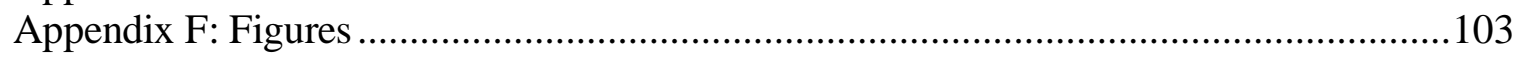




\section{List of Figures}

Figure 1. Integrated model of response to sport injury .................................................. 103 


\section{List of Tables}

Table 1. Participant demographic information ............................................................ 97

Table 2. Interview questions aligned with the International Sport Coaching Framework ...98

Table 3. Code map of iterations of analysis for Professional Knowledge ........................99

Table 4. Code map of iterations of analysis for Interpersonal Knowledge .......................101

Table 5. Code map of iterations of analysis for Intrapersonal Knowledge ......................102 


\section{SUPPORTING THE INJURED ATHLETE: COACHES’ PERSPECTIVES}

\section{ON PROVIDING SOCIAL SUPPORT}

Approximately one million injuries were sustained by athletes participating in sport in the National Collegiate Athletic Association (NCAA) between 2009 and 2014 (Kerr et al., 2015). Injury is an inevitable reality when participating in sport (Chalmers, 2002), and coaches must be prepared to help athletes through injury rehabilitation. Initial evidence suggests coaches believe their primary role during their athletes' injury rehabilitation is helping them return to competition (Podlog \& Eklund, 2007b). More specifically, Fernandes et al. (2014) highlighted the importance of coaches taking a holistic approach to improving injury outcomes for athletes, extending beyond physical rehabilitation to include both social (e.g., helping athletes stay involved with their team) and psychological factors (e.g., managing expectations, referring them to a sport psychology consultant). This holistic approach helps athletes to make a full recovery, ensuring the injury is physically healed and the athlete is psychologically ready to return to sport safely. Social support is one tool available to coaches that helps them to address athletes' emotional, psychological, and social needs related to injury and has frequently been cited as one of the most important psychosocial factors during athletes' rehabilitation (Yang, Peek-Asa, Lowe, Heiden, \& Foster, 2010). Social support is defined as "an exchange of resources between at least two individuals perceived by the provider or the recipient to be intended to enhance the well-being of the recipient" (Shumaker \& Brownell, 1984, p. 13). Johnston and Carroll (1998) found both emotional and informational support from coaches was especially important to athletes as they finished rehabilitation and began re-integrating into practice and competition. Further, Podlog and Dionigi (2010) reported coaches used social support strategies such as goal setting, cognitive reframing, and continued team involvement to improve injured athletes' sense of competence, 
autonomy, and relatedness throughout rehabilitation. Awareness of athletes' needs and knowledge on specific approaches are crucial to effectively supporting athletes whose responses to injury may be highly variable and dependent on the individual.

The integrated model of response to sport injury (Wiese-Bjornstal, Smith, Shaffer, \& Morrey, 1998) offers a framework from which to explore the psychological response to athletic injury. Specifically, the integrated model demonstrates the dynamic nature of injury rehabilitation; both personal factors (e.g., injury severity, injury type, gender, age, athletic identity) and situational factors (e.g., type of sport, coach influence, social support provision, rehabilitation environment) influence athletes' cognitive appraisal of their injury, which informs both emotional and behavioral responses. Within the model, social support and coach influences are recognized as situational factors that can positively or negatively affect how athletes evaluate their injuries. When athletes negatively appraise their injuries, they may experience greater frustration, depression, and anxiety, which can hinder their desire to comply with rehabilitation protocols (Clement, Arvinen-Barrow, \& Fetty, 2015; Johnston \& Carroll, 1998).

Social support research highlights a need for not only a greater amount of support being offered by coaches, but also better-quality support as well (Abgarov, Jeffery-Tosoni, Baker, \& Fraser-Thomas, 2012; Ruddock-Hudson, O’Halloran, \& Murphy, 2012; Ruddock-Hudson, O’Halloran, \& Murphy, 2014). In one study on college athletes' injury experiences, athletes reported their coaches doubted the severity of their injuries and that discussing their injuries with their coaches was challenging (Abgarov et al., 2012). Similarly, Ruddock-Hudson et al. (2012) found professional footballers reported a lack of support from coaches and desired more interactions with coaches during rehabilitation. Additionally, these athletes described their coaches as pushing them to return to play rather than taking the time to rehabilitate. In the 
Ruddock-Hudson et al. (2014) study of professional footballers, the athletes reported coaches offered support immediately following the injury, which dramatically declined once athletes began rehabilitation. Although these studies suggest coaches do not offer support that is both needed and desired by athletes during injury rehabilitation, there are a dearth of solutions for improving these circumstances. Perhaps some of the frustration athletes experienced comes from the difference between their expectations for how their coaches should support them and what coaches are truly capable of providing. For example, Ruddock-Hudson et al. (2012) found a small number of the athletes in their study recognized coaches need to balance simultaneous and competing demands, such as coaching their team while supporting their injured athletes. A critical need exists to examine how coaches perceive the provision of social support to injured athletes and how their coaching knowledge may impact their willingness and ability to do so effectively. Maurice, Kuklick, and Anderson (2017) proposed using the International Sport Coaching Framework (ISCF; ICCE, 2014) to examine how coaches use specific forms of coaching knowledge to navigate athletes' injury experiences, specifically regarding their attitudes and behaviors towards providing social support during athletes' rehabilitation.

\section{International Sport Coaching Framework}

The ISCF was developed by the International Council for Coaching Excellence (ICCE, 2014) and highlights the knowledge effective coaches should possess to promote positive athlete outcomes, such as competence, confidence, connection, and character (Côté \& Gilbert, 2009). Knowledge is categorized into three types: professional, interpersonal, and intrapersonal.

Professional knowledge refers to tactical and procedural understanding (Cassidy, Jones, \& Potrac, 2009), which may include providing information about the injury or specific activities athletes can engage in that will not aggravate their injuries further. Professional knowledge may also 
assist coaches in referring athletes to other professionals who can help. Interpersonal knowledge refers to the social aspect of coaching (Bowes \& Jones, 2006). Coaches can implement interpersonal knowledge with their injured athletes to gain a better understanding of the social support needs of their athletes, which are dependent upon the type of stressor experienced (i.e., optimal matching hypothesis; Cutrona, 1990; Cutrona \& Russell, 1990). Social support from coaches that is misaligned with athletes' needs can be perceived by athletes as non-supportive and represent a poor use of interpersonal knowledge. Lastly, intrapersonal knowledge refers to coaches' ability to reflect and be introspective (Côté \& Gilbert, 2009). Coaches can use intrapersonal knowledge to improve their interactions with injured athletes and help create a safe and positive environment for athletes to recover from their injuries. Despite the reality that injury is an inevitable facet of sport (Chalmers, 2002), there is a lack of research examining coaches as a component of the rehabilitation experience. By using the ISCF and its three knowledge types as a framework, researchers can begin to gain insight into coaches' expectations and experiences with injury and potentially offer explanations for why athletes have felt unsupported by their coaches (Maurice et al., 2017).

\section{Coaches' Provision of Social Support}

Several studies have examined how coaches have engaged with their injured athletes during their recovery. In a study of eight professional coaches of high-performance athletes (i.e., national to Olympic level), Podlog and Dionigi (2010) found coaches acknowledged the difficulties their athletes faced (i.e., re-injury anxiety, lack of confidence or time pressure). The coaches also identified strategies that helped them support injured athletes, such as forming a treatment team, having open communication, and offering social support. Coaches within this study expressed the value of social support as a critical component of the recovery process for 
their athletes and a need to take a more individualized approach to help athletes return to practice and competition (i.e., using interpersonal knowledge). Within the provision of social support, coaches needed to not only tailor their approach to their specific athletes (i.e., interpersonal knowledge), they also helped the athletes adjust and manage their training plans (i.e., professional and intrapersonal knowledge). Though the professional coaches in this study appeared to be incorporating elements of the ISCF coaching knowledge when working with their injured athletes, these coaches represent the behaviors of only eight coaches at an elite level of sport and these views may not transfer to coaches' views at other levels of sport (e.g., high school, college).

Further, evidence suggests a discrepancy exists between the degree of social support coaches believe they provide and the amount of social support injured athletes report receiving from coaches (Corbillon, Crossman, \& Jamieson, 2008; Gould, Udry, Bridges, \& Beck, 1997; Udry, Gould, Bridges, \& Tuffey, 1997). For example, some professional coaches have explained their primary role in injury rehabilitation is to prepare athletes for a return to competition (Podlog \& Eklund, 2007b) while athletes have expressed that coaches frequently leave them to make their own decisions about how to best return from injury (Udry et al., 1997). Injured athletes who are without social support during their rehabilitation may experience higher levels of depression (Clement \& Shannon, 2011), and athletes who are dissatisfied with the social support they received are likely to experience psychological distress (Green \& Weinberg, 2001). Udry et al. (1997) interviewed members of the U.S. Ski Team who had sustained season-ending injuries and found these athletes perceived their coaches to be the most negative influence during their rehabilitation period. Specifically, these athletes felt ignored during their recovery as coaches reduced or stopped communication with them entirely. In other instances, the athletes 
felt coaches were insensitive to injuries and reported coaches thought they were a problem and did not believe fully in the extent of injuries. Additionally, the athletes explained their coaches did not express confidence in their ability to return from injuries and were often unavailable to help during rehabilitation, which left the athletes to manage their recovery on their own. This study, however, focused solely on the interpretations of the athletes without giving voice to coaches. Many of the studies reporting a lack of social support from coaches (e.g., Abgarov et al., 2012; Ruddock-Hudson et al., 2012; Ruddock-Hudson et al., 2014) have not included coaches. By focusing solely on athletes, researchers cannot fully explain why coaches do not offer social support that athletes find beneficial.

Injured athletes have reported seeking out and receiving social support most frequently from their athletic trainers (Clement \& Shannon, 2011; Robbins \& Rosenfeld, 2001; Yang et al., 2010), which may be due to the quantity of time spent with athletic trainers during injury recovery or the perceived unavailability of coaches who are focused on participating athletes (Ruddock-Hudson et al., 2014). When athletes have reported receiving support from their coaches, they most frequently cited their coaches as providing informational support (Johnston \& Carroll, 2000; Johnston \& Carroll, 1998; Corbillon et al., 2008; Rosenfeld, Richman, \& Hardy, 1989). Informational support may include education about the injury, feedback about their return timeframe, or their role on the team. Injured athletes have reported coaches do not care about their recovery and remain distant while the injured athletes recover (Udry et al., 1997), which suggests coaches may not be offering enough emotional support. Tangible support may be more difficult for coaches to provide due to organizational restrictions (i.e., NCAA). Robbins and Rosenfeld (2001) suggested, in some cases, coaches did not offer extra support for injured athletes because it may be perceived as unfair to the rest of their team, even if coaches were 
aware that the addition of support would have been helpful. Thus, it appears essential for coaches to help injured athletes create a network that includes many people who can meet their diverse social support needs.

\section{Integrating Coaching Knowledge into Injury Rehabilitation}

For athletes rehabilitating an injury, maintaining a connection to their coaches is an essential part of progressing effectively through the stages of rehabilitation (i.e., occurrence of injury, rehabilitation, return to competition) and returning to competition (Bianco, 2001; Podlog \& Dionigi, 2010). In the initial injury stage, athletes who sustain an injury first experience an emotional response characterized by uncertainty about the remainder of their season or their ability to continue participating in their sport (Johnston \& Carroll, 2000; Madrigal \& Gill, 2014; McDonald \& Hardy, 1990). Using interpersonal knowledge, coaches can be a source of emotional support for athletes and assist athletes in maintaining their connections to the team. In the second stage, athletes receive a diagnosis and begin their rehabilitation (Bianco, 2007). Athletes rehabilitating an injury are susceptible to feelings of grief (Evans \& Hardy, 1995; Tracey, 2003) and low self-esteem (Deroche, Stephan, Brewer, \& Le Scanff, 2007) as they are now physically removed from their sport environment. Coaches, then, may find time becomes a barrier as they attempt to engage with injured athletes who are not active in practices and games. Coaches may have the opportunity to demonstrate their professional knowledge as it relates not only to the specific injury but also to the injury recovery process. However, it can be argued offering specific information on the injury itself is outside the bounds of coaching competency and perceived as a barrier by coaches. Finally, in the third stage, athletes finish their rehabilitation and return to their sport. During the last phase, athletes can experience doubts about their physical competence and negative psychological responses, such as re-injury 
anxiety (Houston, Cross, Saliba, \& Hertel, 2014; Podlog \& Eklund, 2007a). Coaches can use intrapersonal knowledge by engaging in reflection with injured athletes to highlight the effort put in and the progress athletes have made throughout the process of rehabilitation. Due to the degree of influence coaches have on their athletes, there is great potential for coaches to actively support athletes throughout the stages of rehabilitation by using various coaching knowledge types and promoting positive outcomes (Bianco, 2007).

\section{Gaps, Limitations, and Study Purpose}

Coaches may be under the impression they are already providing social support to their injured athletes (Podlog \& Dionigi, 2010), but as research has shown (e.g., Abgarov et al., 2012), athletes are not satisfied with the support coaches are providing. Dissatisfaction with coaches' quantity and quality of social support has the potential to create negative consequences for injured athletes. Much of the research on social support for injured athletes has been from the perspective of the athletes and has not included coaches (e.g., Abgarov et al., 2012; Corbillon et al., 2008; Ruddock-Hudson et al., 2014). The research that has examined coaches' perspectives on injury rehabilitation (e.g., Podlog \& Eklund, 2007b; Podlog \& Dionigi, 2010) was completed almost a decade ago and did not use a framework specific to coaching. There also remains a dearth of literature on coaches at the collegiate level, which represents a competitive environment dependent upon preventing injuries and returning athletes to play quickly. In National Collegiate Athletic Association (NCAA) Division I (DI) sports, arguably the most competitive of any collegiate sport division, the pressure placed on athletes can be as intense as some professional sports leagues (Beron \& Piquero, 2016; Huml, 2018). NCAA DI athletics are operated like a business despite athletes being labeled as amateurs and receiving little compensation in return for the stressors they endure, such as injury (Sanderson \& Siegfried, 2018). In the business of college athletics, coaches' 
jobs often depend on their teams' success. It is in the interest of college coaches, and their athletic departments, to keep their athletes healthy to produce the best results. Given the unique context of NCAA DI sport, it is vital to create environments that both support athletes' well-being and allow coaches to use their knowledge and experiences to support their athletes. Until college coaches have been studied, researchers and those involved in college athletics will continue to have an incomplete understanding on how coaches can effectively interact with their injured athletes.

The purpose of this study was to explore collegiate coaches' knowledge related to the provision of social support to injured athletes throughout the phases of rehabilitation and return to competition. Using the ISCF as a guiding framework, this study had three primary goals: (1) gain insight into how coaches perceived their role in providing social support throughout the injury recovery process, (2) explore how coaches used the ISCF knowledge types in the context of supporting injured athletes, and (3) explore what coaches perceived to be barriers to their delivery of effective social support.

\section{Methods}

\section{Researcher Positionality}

The researcher's epistemology is that of a social constructivist. Social constructivism recognizes the impact culture and context have on an individual and how they experience the world and create their own meaning (Crotty, 1998; Fish, 1990). Social constructivists do not see an absolute truth, but rather they acknowledge all meaningful realities are socially constructed and influenced by culture (Crotty, 1998). The participants in this study were examined, not with an absolute truth of what is right and wrong, but with an appreciation for their culture and context to gain insight into how this shaped their views of coaching injured athletes. The 
researcher recognized that the coaches' reality and her own reality are the result of social processes and are limited to the context in which they were experienced.

\section{Study Design}

A generic qualitative approach, also referred to as an eclectic design, was used to explore coaches' perceptions regarding their roles in the injury recovery process, the ways they use the ISCF knowledge types, and barriers they perceive in supporting their injured athletes. The exploratory approach needed to address the purpose of the current study does not fit neatly into one specific methodology. Merriam (1988) described studies using the generic qualitative approach as those that "simply seek to discover and understand a phenomenon, a process, or the perspectives and worldviews of the people involved" (p. 11). Percy, Kostere, and Kostere (2015) described the need for generic qualitative approaches when "the researcher has a body of preknowledge/pre-understandings" about the topic in question that the researcher "wants to be able to more fully describe from the participants' perspective" (p. 78). In the case of the current study, the researcher has knowledge of the research suggesting that athletes do not feel fully supported by their head coaches and is using this exploration to gain the perspective of head coaches and the ways they use their coaching knowledge in the context of athletic injury. Generic qualitative approaches also seek to gain a broader range of experiences from a more diverse sample, supporting the current study’s focus on maximum variation sampling (Percy et al., 2015). According to Cooper and Endacott (2007) and Caelli, Ray, and Mill (2003), generic qualitative approaches should have an explicit focus on their theoretical position to enhance the rigor of the studies. As a social constructivist, it was important for the researcher in the current study to focus on how coaches used their ISCF knowledge types within their unique context and culture. Thus, a generic qualitative approach was most appropriate for this inquiry. 


\section{Participants}

Participants were 13 NCAA DI head coaches $\left(M_{a g e}=45.85, S D=11.2\right)$ including five women $\left(M_{\text {age }}=38.6, S D=12.7\right)$ and eight men $\left(M_{\text {age }}=50.4, S D=7.87\right)$ from 13 DI universities in the United States. Five coaches ( 2 female, 3 males) came from mid-sized universities (student population of 3,000-9,999) and eight (3 females, 5 males) from large universities (student population of at least 10,000$)$. Four coaches were from universities without a football program (2 female, 2 male), four from universities with football programs in the Football Championship Subdivision (1 female, 3 males), and five from universities with football programs in the Football Bowl Subdivision (2 females, 3 males). The participants' head coaching experience at the DI level ranged from 1 to 23 years $(M=9.1$ years $)$ and four to 31 years $(M=18.5$ years $)$ in any NCAA division. The sports coached by men within this sample included men's and women's basketball, baseball, football, women's golf, women's ice hockey, and men's and women's tennis. Sports coached by women within this sample included men's and women's swimming and diving, women's gymnastics, women's rowing, women's soccer, and women's volleyball (see Table 1 for more demographic information).

The coaches in this study had experience in coaching athletes who had sustained an injury and returned to participation within the past year. The injured athlete must have sustained at least a minor injury causing time-loss from their sport (e.g., 4-7 days; Hagglund, Walder, Bahr, \& Ekstrand, 2005), defined as “a physical complaint resulting from sports participation that forces the athlete to interrupt or modify his usual training plan for at least one training unit" during their competitive season (Malisoux, Frisch, Urhausen, Seil, \& Theisen, 2013, p. 2896). Coaches were excluded from the study if their most recent athlete injury experience occurred more than one year ago, their athlete did not return to competition following an injury, or if their 
only experience with injury was a head injury, season-ending, or career-ending injury. Head injuries were excluded from this study due to differences in athletes' cognitive and emotional responses compared to those experiencing musculoskeletal injuries (Hutchison, Comper, Mainwaring, \& Richard, 2011; Hutchison, Mainwaring, Comper, Richards \& Bisschop, 2009).

\section{Procedures}

After receiving Institutional Review Board approval, participants were contacted for recruitment using a purposive sampling approach. The researcher used the United States Census Bureau's regions to seek out maximum variation within the sample. There are nine regions total (Pacific, Mountain, South Atlantic, Middle Atlantic, New England, West South Central, East South Central, West North Central, and East North Central) that were used to identify NCAA DI coaches for participation. The researcher contacted 188 head coaches across the nine regions via email with information about the study and details for inclusion criteria if they wished to participate. The researcher attempted to contact the selected coaches via email and telephone up to three times in a 10-day period before moving onto another coach in the region. The number of coaches contacted per region ranged from two to 68 . Due to their low numbers, female coaches were identified and contacted first and in the following order: those who coached men's teams, co-ed teams, and women's teams. From there, male coaches were identified and contacted to achieve maximum variation within the sample (e.g., varied ages, sport and gender of athletes coached). Using the maximum variation sampling strategy to achieve a heterogeneous sample was desirable in this study because of the dearth of research on coaches and injury; it was important to first gain the perspective of a diverse group who had varied coaching experiences rather than focus on one specific demographic of coaches. Interviewing the sample of coaches within this study using the maximum variation sampling strategy allowed the researcher to 
uncover a broader spectrum of coach experiences (Miles et al., 2014). The final sample included 13 coaches who volunteered to participate (6.9\% response rate); all met eligibility criteria.

Coaches who agreed to participate in the study were sent a cover letter detailing what their participation would entail and how they would be compensated for their time (i.e., coaching book). The researcher conducted interviews lasting 10 to 57 minutes over the phone or a video chat service, such as Skype, which were audio-recorded. The mode of communication for each interview was determined by the participant based on their interest and comfort level. Eleven of the coaches were traveling to practice or competitions while being interviewed and chose to be interviewed over the phone.

Data were collected using a semi-structured interview guide (Bellamy, Ostini, Martini, \& Kairuz, 2016; Mertens, 1998). The semi-structured interview guide provided the researcher with flexibility to probe participant responses further while allowing her to maintain a level of consistency throughout her participant interviews. Each interview began by developing rapport with the coaches and building trust by explaining the researcher's interest in giving coaches a voice in the literature as well as describing her family's history in coaching. The interview questions were developed based on the core components of the ISCF, addressing multiple knowledge types (i.e., professional, interpersonal, and intrapersonal knowledge), and the stages of rehabilitation (see Table 2 for interview guide mapped onto the ISCF). Social constructivism shaped the format of the interview questions to elicit responses from coaches that would provide insight into both their specific social context and how their interactions and experiences shape their understanding of their world (Cushion, 2011; Crotty, 1998). The audio recordings and transcripts were kept in Filelocker, a software service that encrypted the files and protected participant confidentiality. The participants were reminded at both the start and close of the 
interviews their confidentiality was of the utmost importance to the researcher. Participants were given the opportunity to choose their own pseudonyms so their identities may be protected in any representation of the data. Data collection spanned several months, throughout which the data was also transcribed and coded.

After interviews were transcribed and the researcher began coding the interviews, participants were contacted again to engage in member reflections (Smith \& McGannon, 2017). Three of the 13 coaches responded to the researcher's follow-up to conduct member reflections. Member reflections were used to create a shared dialogue between the participant and the researcher about the analysis of the results. The member reflections allowed the participants to ask questions about the interpretation of the data as well as opportunities to evaluate and provide feedback and on the researcher's findings (Tracy, 2010). The member reflections were a coparticipatory process that furthered the researcher's understanding of the data. During this conversation, the coaches were also given the opportunity to add to the information they provided in their initial interview. The researcher prompted the participants with member reflection questions, which provided the participants an opportunity to give an opinion after hearing the researcher's interpretations and shape the emerging analysis (Tracy, 2013). For example, after explaining her interpretation of the coaches' perceptions of athletes as barriers to coaches' delivery of social support, the researcher asked the participants what they thought of the interpretation and what that means to the participants in their specific context (i.e., within their team). The member reflections were not about verification but rather were intended to further the researcher's interpretations of the findings and yield further insight. From these member reflections, the researcher learned that the coaches found value and meaning in the researcher's interpretations of the data (Tracy, 2013). Conducting member reflections rather than member 
checks allowed the researcher to engage in both epistemological constructionism (i.e., as knowledge is socially constructed, knowledge is not free from theory and understandings prior to engaging with participants) and ontological relativism (i.e., the perception of reality is dependent upon the mind experiencing it) (Smith \& McGannon, 2017), which complemented her social constructivist epistemology. Upon the completion of member reflections, all coaches who participated in the study were given a coaching book in appreciation of their volunteer participation.

\section{Data Analysis}

The current study used the ISCF knowledge types as a guiding framework to conduct a thematic analysis of the data. The researcher first familiarized herself with the data by listening to the audio recordings of the coach interviews and reading the transcripts for accuracy and familiarity. While engaging with the data, the researcher created analytic memos (Miles, Huberman, \& Saldaña, 2014) to denote interpretations of the data from collection through analysis. The interpretations noted in the memos pertained to how interview responses aligned with components of the ISCF and initial patterns within each coach's unique context (i.e., sport, experiences). As a social constructivist, it was of value to take note of how coaches explained, and ascribed meaning to, the culture of their team, such as the way coaches engage differently with male and female athletes.

The ISCF knowledge types were used as a guide for coding, which occurred in two cycles: (1) deductive in vivo coding and (2) focused coding. Deductive in vivo coding was selected as the method of analysis over inductive coding because of the need to identify codes connected to the existing framework (i.e., ISCF). The researcher used deductive in vivo coding during the initial coding process to identify the presence or omission of the ISCF knowledge 
types. In vivo coding is appropriate when the researcher wants to honor the voices and the language used by the participants (Saldaña, 2013; Stringer, 1999). As a social constructivist, it was essential for the researcher to select a coding approach focused on the language used by the participants to better understand their lived experiences, such as the way they addressed concepts like perceived responsibility in their role as the head coach or descriptions of relationships with their athletes. Saldaña (2013) explains that in vivo coding allows a researcher to highlight the most significant ways the participant speaks about a phenomenon by coding components such as action verbs, impactful nouns, clever phrases, and/or repetition of words and phrases. One participant was analyzed separately as an extreme case due to distinct dissimilarities in the interview content and process as compared to the remaining participants. These distinctions are examined and described in further detail at the end of the results section. First cycle coding resulted in a list of codes related to each of the ISCF knowledge types.

Second cycle coding consisted of the method of focused coding (Charmaz, 2006), which allowed the researcher to use the most frequent and salient codes to create categories, subthemes, and themes related to how coaches perceived their roles in the recovery process, the ways coaches used their ISCF knowledge types to support their athletes, and the barriers perceived by coaches as impeding their delivery of effective social support. A detailed map of these iterations is provided in Tables 3 through 5. During this process, the researcher continued to construct her understanding of the lived experiences of the coaches and the ways they made sense of their interactions. To enhance credibility, critical friends (Smith \& McGannon, 2017) were used to process the researcher's biases after she created codes, sub-themes, and themes from the data. The use of critical friends allowed for varying perspectives to view the data and were used for “challenging and developing interpretations made by any one researcher as they construct, not 
find or discover through consensus, a coherent and theoretically sound argument to construct, support and defend the case they are making" (Smith \& McGannon, 2017, p. 13). The researcher used two levels of critical friend engagement throughout the analysis. The first critical friend has experience in qualitative inquiry and has conducted research on the psychology of injury; her role as a critical friend began when the sub-themes and themes were first developed by the researcher. This individual challenged the researcher's interpretations of the data to consolidate, clarify, and organize the sub-themes and themes. The second critical friend has experience in qualitative research and the coaching literature. This individual was engaged following the development of an initial theme structure and challenged the researchers' interpretations to achieve additional theme refinement, clarity, and parsimony (e.g., Are these ideas distinct? What makes them so? How might this theme be re-labeled to more clearly reflect the contents of the data?). Each critical friend was used to clarify the researcher's process and basis for interpretation, including impressions and interpretations. Both critical friends and the researcher maintained records of these conversations for the audit trail and later reflection by the researcher.

\section{Results}

The results are organized according to the ISCF knowledge types (i.e., professional, interpersonal, and intrapersonal), which were used as a framework for the analysis. Themes and subthemes are described within each knowledge type with key quotes from coaches.

\section{Professional Knowledge}

Five themes characterized coaches' demonstration of professional knowledge in the context of athletes' injuries: (a) using resources, (b) injury prevention, (c) phases of injury rehabilitation, (d) sustaining injured athletes' involvement, and (e) athlete welfare. See Table 3 for the iterations of analysis for professional knowledge. 
Professional knowledge of resources. The ability to recognize when to use a resource, and which resource is the most appropriate, is an important demonstration of professional knowledge as it relates to injured athletes. Relative to professional knowledge of resources, two sub-themes were identified: (a) the network roles that need to be fulfilled to support injured athletes and (b) the accessibility of resources for supporting injured athletes.

Within the first sub-theme, coaches described the network of individuals needed to fully support their injured athletes, such as doctors and sport psychology consultants. All 13 coaches emphasized the importance of having a network of professionals (e.g., athletic trainers, doctors, and sport psychologists) as resources to help them support their injured athletes. Howard, a baseball coach, described the need to "rely on the medical professional. And most importantly, for Division I, or any coach, you have to listen to the medical professional." Howard further expressed:

As a coach, I have no medical expertise; none. I understand baseball and I understand movements related to baseball and things that need to happen, but structurally and medically, I don't, so I always lean on professionals to guide me if I'm unaware. Many of the coaches relied on athletic trainers, sometimes described as medical professionals, as their primary resource when athletes sustained injuries and expressed the value of using their athletic training staff when designing training plans. All the coaches discussed the importance of listening to their athletic trainers and trusting they have athletes' best interests in mind, as demonstrated by women's soccer coach, Lauren:

Listen to your medical staff, if they're telling you to do something, don't overrule them. As much as sometimes I want to strangle my AT, she's not trying to sabotage us and she's 
not trying to control my decisions, she's trying to give me all the information I need to make the best one.

The coaches also addressed the value of sport psychology consultants as members of a support team. All the coaches reported having access to sport psychology services, but Claire (gymnastics), Kevin (women’s basketball), Lauren (women's soccer), Noah (men’s and women's tennis) and Owen (men's basketball) were the only coaches making referrals for these services. Coaches who made referrals to sport psychology services understood the role sport psychology has during recovery, as Noah, a men's and women's tennis coach, explained:

If you have somebody in your department to refer them to where they can get that help, because many times it's not the injury that's affecting the player after they're back on the fields or the courts, it's the psychological hurdle that they have to get over.

Within the second sub-theme, coaches described the accessibility of resources for supporting injured athletes. For some coaches, the lack of medical resources available impacted their ability to find support for their athletes (e.g., informational support for their injury). Noah (men's and women's tennis) discussed this in his interview, "we were under-equipped in the sports medicine department. Very small room, very few people working in there to service the sport. So, a lot of times we couldn't get in to see him when we needed to.” David (women's soccer) reported, "we don't have that overall access over a 12-hour day to all facilities, where we can schedule what works for us so that we can maintain that strength so that we limit some of those injuries." The way resources were allocated varied based on the sport and athletes' status on the team. Mason (football) explained: "I guarantee, the number 10 guy would get [the MRI]. And the number 101 guy would wait until it was paid for by their insurance, which I think is wrong." Coaches, such as Noah (men's and women's tennis) and David (women's soccer), were 
attempting to use their professional knowledge to support their athletes, but were limited to the resources available to them.

Professional knowledge of injury prevention. Relative to the theme of professional knowledge of injury prevention, coaches described the importance of using and staying current on proper training techniques and helping athletes with the mental aspects of injury. Many coaches noted a lack of medical knowledge but were knowledgeable of injury prevention (e.g., fatigue, poor training) and applying instructional techniques to reduce injury incidence. For example, Lauren (women's soccer) explained, “we are very strict on teaching correct technique because although it doesn't completely eradicate injury, it minimizes it and there's plenty of research on that." David (women's soccer), expressed his concerns about his athletes' being fatigued during competition:

I know that the longer you leave someone in a situation where they start to fatigue, the more likely it is that they're going to pick up an injury, because they can't play at the same level when they get fatigued as they did when they first went into a game. Many of the coaches felt prepared and educated on how to use preventive approaches to injury, such as addressing training techniques, but felt less knowledgeable about what to do after the injury was sustained. The coaches shared candidly that their medical knowledge regarding injury is limited but they expressed a desire to continue to learn more and educate themselves about their athletes' injuries, as expressed by Tanya (women's volleyball):

I am very candid about what I don't know and I have no problem asking our athletic trainers just, "Wow, I've never seen this before. I don't even know what you're talking about with this injury. So, can you explain it to me? Because I don't know." 
Emily (women's rowing), however, had stopped trying to learn more about injuries: “I'm not allowed to do stuff with them [injured athletes]. I have to stay hands off...But as far as me looking for more information, I've stopped doing that here because they [the university] discourage us from doing that." Emily's situation appeared to be unique to her university as this was not expressed by any other coach.

Professional knowledge on phases of injury rehabilitation. Professional knowledge in the athletic injury context requires coaches to be aware of the phases of injury rehabilitation and the ways athletes' needs may vary across each phase. Relative to professional knowledge of the phases of injury rehabilitation, two sub-themes were identified: (a) managing their own emotional responses to athletes' injuries and (b) being cognizant of performance decrements that may occur as a part of the recovery process.

Within the first sub-theme, coaches described a need to monitor their own emotional responses during the occurrence of injury phase of rehabilitation. Coaches recognized their reactions to athletes' injuries often impacted their athletes' reactions. Coaches were asked to describe how they engage with athletes throughout the phases of rehabilitation (i.e., occurrence of injury, treatment and recovery, and return to full competition). Lauren's (women's soccer) explanation of her reaction to injuries was like other coaches, where staying calm herself was a necessary element of keeping her athlete calm:

I try to be very calm and try and just reiterate, 'Hey, it's part of the game. You're not the first, you're not the last.' I just try and take the emotion out of it, because it's a very emotional time.

Claire, a women's gymnastics coach, explained the importance of managing emotional responses based on injury severity: 
If it's something that happens in practice and it's a one-time impact kind of injury, we obviously go to the athlete right away and give them the care that they need. If it's an over time kind of thing, we usually check in periodically, and work with them on what makes the most sense for them to do that day, whether it's rehab, or if they have to alter practice in any way.

Within the second sub-theme, coaches described the importance of acknowledging the ways athletes' performance may decline because of their inability to participate in practice and training during the treatment and recovery phases of rehabilitation. Owen (men's basketball), noted:

Yeah, I think the main thing when those guys get hurt is when they come back, their conditioning is not as good as it was before they left. And so physically they struggle a little bit just kind of getting their bodies back on a little bit.

Noah (men's and women's tennis) echoed this sentiment:

We know that they're more than likely not going be hitting exactly like they were, or performing exactly like they were before the injury, based on how long it was. And we also know that they could get extra critical, or disappointed, or negative towards their game, when it's not coming right back.

Professional knowledge on injured athletes' sustained involvement. Relative to professional knowledge on injured athletes' sustained involvement, all the coaches stressed the importance of keeping athletes involved with the team after they have become injured. Coaches described strategies they use to incorporate their injured athletes into practice. Doing this allowed athletes to maintain contact with their team and for some, get physical conditioning. The 
coaches shared a belief that being around the team would keep athletes involved with the team's progress. Kevin (women's basketball) explained:

I want them to be at practice and I want them around the team. Just not be away from them, so I expect them at practice... I make them take notes, make sure they know what's going on so they don't get behind.

Some coaches provided challenges for injured athletes to keep them engaged, such as Claire (women's gymnastics):

We've given one of our athletes a challenge, and she needs to reach out to three different people during practice, and encourage them. So, giving them ways that they can become really good teammates during this time and not all the focus is just on the rehab... I think that's really key for getting them mentally out of the struggles of the injury.

Coaches described the value of maintaining a social connection to their teammates by giving injured athletes new roles on the team. Coaches shared their technique of assigning injured athletes new roles to keep them engaged in practice, such as Lauren (women's soccer):

A big help has been just giving them other roles, so they have a specific role at practice. So even if it's things like, "Hey, I need the waters. Can you help me set practice up?" Go through the practice plan with them, just so they feel like they're part of it.

Ultimately, coaches seemed to prefer athletes do their rehabilitation exercises during team training times to still be involved in the team activities.

Professional knowledge on athlete welfare. Coaches expressed the importance of keeping their players safe. Relative to professional knowledge on athlete welfare, two subthemes were identified: (a) how to make the right decision about return to play and (b) having a person-first focus. 
Within the first sub-theme, coaches described the importance of trusting both their athletes and medical staff regarding readiness to return. When it came to making decisions about returning to play, coaches frequently described the need to keep their athletes' welfare in mind. Howard, a baseball coach, shared:

My responsibility is to have a good understanding of each player's ability to deal with things...And like I said, we got measures in place to protect these young kids and make sure they're not in any danger, and hopefully, we'll continue to monitor and keep those types of things from happening.

In some cases, coaches had to sit down with athletes and remind them to focus on recovering, like Allison, a men's and women's swimming and diving coach:

A true athlete's going to want to, after an injury, get back as soon as possible, but you have to be willing to say, "No, you're not ready," and say, "Just give it another week or two weeks." And that's kind of what you have to do with those ones.

Tanya (women's volleyball) explained:

I think it's a huge responsibility of the coach to acknowledge that and really dial back and figure out a way to progress that player back, where it's not rushing them and yet still pushing them... I think it's our responsibility as the coach to really dictate how we get them back without stressing them out, feeling, "Oh my gosh, I have to get back this quickly, I need to be back on the floor, my team needs me." All of that, I think, it's our responsibility to keep the athletes safe.

Within the second sub-theme, coaches described a need to focus on their athletes as a person and not on their win-loss record while their injured athletes recover. Allison (men's and 
women's swimming and diving) extended the notion of keeping athletes safe and treating them as people:

Know at the end of the day, they [athletes] and their well-being and them...They as a person, is more important than your win-loss record, and I think sometimes coaches get caught up in that and I know there's all different levels and your job is on the line as a coach and all these things, but you will be remembered far beyond your win-loss record as compared to how you're treating your student-athletes.

Several of the coaches reflected on the need to keep athletes' long-term health in mind beyond the current season, such as David (women's soccer): "I definitely don't want somebody to have something that happens and then later in life they can't be pain free as a mom or grandmother." Mason (football), also focused on thinking of athletes as people first:

And that's one of my big things is, I think everybody needs to realize, and especially the training room, players only have $\mathrm{X}$ amount of games. Most of them aren't going to play professional football. Football ends. You can play rec basketball, you can play rec baseball, you can play adult baseball and all those things until you're whenever. Football ends. You have 44 college games, let's say. And when you start taking games away, the player needs to know that that's what's going to happen.

David (women's soccer) spoke to the need for a person-first focus as well:

I think those coaches out there who take the time to invest in their student athletes, I say whether you ever win a championship or not, you're going to find out over the course of your career that you won...And whether they're injured, whether they're being the superstar on the field, whether they're the last person off the bench, shouldn't matter. If 
you're invested, invest in the whole person, right, because, to me, that's where the reward comes from.

\section{Interpersonal Knowledge}

Two themes were identified related to how coaches demonstrated interpersonal knowledge in the context of athletic injury: (a) communicating with injured athletes and (b) perceiving athletes as barriers. See Table 4 for the iterations of analysis for interpersonal knowledge.

Interpersonal knowledge on communicating with injured athletes. Communicating effectively with athletes is a critical part of demonstrating interpersonal knowledge. Relative to interpersonal knowledge on communicating with injured athletes, two sub-themes were identified: (a) using communication effectively to support injured athletes and (b) challenges in communicating with injured athletes.

Within the first sub-theme, all 13 coaches described the importance of keeping open lines of communication with their injured athletes. Owen (men's basketball) explained:

You always want to try to spend more time with your guys and have a great communication line with those guys whether they're injured or not injured. So, I would say that's one thing that I think all head coaches want to try to do a better job of. You never can do too much of that.

Howard (baseball), described the importance of focusing communication on athletes' feelings during recovery:

The dialogue is how they're feeling, what they're feeling, and the responses to where they would be normally and where they're at currently....Sometimes they want to do more and 
that can be harmful. So, it's a steady course of contact, maintaining good dialogue, obviously wanting them to get healthy sooner rather than later, both me and the player.

Women's gymnastics coach, Claire, echoed these sentiments:

We really just check in with the athlete, because everyone is so different in what's holding them back, so we really just try to sit down, and talk with them, and figure out where they're lacking, whether it's their confidence, whether they're nervous about competing, or the fear of failure, fear of letting their team down, figuring out where they're struggling, and then try to address it from there.

Wyatt (women's ice hockey) explained, “you're going that extra mile for them, even if it's a text message at nine o'clock at night, 'Hey, how'd today go?'” According to Owen (men's basketball):

Even though you're a mentor the whole time, I think during those times you become even more so of a mentor than a coach to them, by just trying to talk to them and figure out how they're feeling, how they're doing and just sharing some lighthearted conversation with them instead of it just being about basketball or whatever sport you're coaching. I think the best thing is just try to spend time with them, make sure they don't close themselves off from the group.

Within the second sub-theme, coaches described challenges associated with dividing focus between the injured athletes and the rest of the team. Kevin (women's basketball) shared: I can't stop everything and just worry about the injured player, so maybe an injured player doesn't feel that they're wanted or I care about them as much because I have all these other athletes that I have to deal with.

Claire (women's gymnastics) highlighted: 
It's so common to, I don't want to say ignore those athletes, but they're not doing what everybody else is, and so you're not working with them one-on-one like you are the rest of your team. I think finding the opportunity to go, and interact with them, and socialize with them during the practice is important, so they feel like they're still part of it.

The coaches also discussed receiving feedback from their athletes about how injuries were handled, and many reported improving their approach in response. Lauren (women's soccer), shared: "what our athletes have reported to us when they're injured, is sometimes we can disconnect from them and then they think we don't care about them so we've tried to do a better job with that this year." To improve communication, coaches discussed the importance of asking for and incorporating athletes' feedback into their coaching practices.

Although aware of the need for improvements in communication, two of the coaches also discussed communication challenges not specifically addressed within the interview guide.

Wyatt (women's ice hockey) and Noah (men's and women's tennis) both discussed the gender of their athletes as a factor in how they communicated with athletes regarding their injury. Wyatt (women's ice hockey) explained:

Women, they get the vulnerability of the injury...They're so much easier to coach in that way. They're more mature in a lot of ways, maybe immature in other ways. Not so much immature, but they're more insecure and I think that comes from society.

Noah (men's and women's tennis) explained:

The funny thing is, my women's teams tend to seem actually more gritty than my men's teams...they play through injury more, where they don't want to go to the trainers and say they're injured unless they're really injured. My men's teams seemingly whined more 
about injuries... When one of them [women] does complain, then I take it real serious because I don't think they're crying wolf.

Noah further explained that the differences he observed between men's and women's responses to injury led him to change his approach to coaching.

Interpersonal knowledge on perceiving athletes as barriers. When asked about the barriers they face when providing social support, coaches agreed the injured athletes themselves were sometimes the biggest barrier. Relative to interpersonal knowledge on perceiving athletes as barriers, two sub-themes were identified: (a) athletes' unrealistic expectations for recovery and (b) athletes' dishonesty.

Within the first sub-theme, coaches expressed frustration with the expectations their injured athletes have regarding the recovery process. Mason (football), explained:

They believe the training room, they're miracle workers and they're not... players want immediacy, and sometimes injuries don't immediately heal. And then you try to come back, and you play too early, and then now you have this lingering injury and now, "Why do I have this still? I thought you guys fixed it." Well, you rushed it and we said you could practice.

Emily shared her frustrations in "dealing with them completely not understanding the process even when you explain it to them." Overall, the coaches felt the expectations the athletes had for the athletic training room and their coaches were at times barriers to helping athletes get and stay healthy.

Within the second sub-theme, coaches expressed frustrations over their athletes' dishonesty about being injured and the extent of their injuries. Noah (men's and women's tennis) reflected: 
I just finally said straight up, "I don't think you're injured." I said, "Your behavior is fitting somebody who's ducking the competition, you're fearing the competition, you don't want the pressure." And then he's, "Oh, you don't believe me? You don't think I'm injured?" So, then I told him, "No, it's the behavior." I said, "I'm confused because you're saying you're injured and then you're sitting out practice, but then I walk up to the courts and you're out there hitting balls with somebody before practice starts, hitting 100 miles an hour on your forehand, but then you sit out the rest of practice, 'cause you're injured." Other coaches felt stressed about the lack of communication and honesty coming from their athletes regarding how they are feeling. Claire (women's gymnastics) reported: “They might tell one person one thing, and a different coach another thing, or they complain about an injury to somebody else, but then they don't tell the coach what's going on." Howard (baseball) reported the key is to continue to "gain the trust and the confidence from the player so that he can tell me exactly what's going on without fear of, 'He's going to take me out."'

\section{Intrapersonal Knowledge}

Two themes were identified related to coaches' use of intrapersonal knowledge to inform their work with injured athletes: (a) reflecting on personal coaching experiences and (b) reflecting on personal athletic experiences. See Table 5 for the iterations of analysis for intrapersonal knowledge.

Intrapersonal knowledge on personal coaching experiences. The coaches addressed a need to reflect and learn from past interactions with their athletes as a coach, a key element of intrapersonal knowledge. Lauren (women's soccer) described her experiences: 
We don't always get it right because, again, that individual difference piece, some kids don't want me anywhere near them. They're like, "Leave me alone." Some kids want me there more than I can be there so we don't get it right every time.

Lauren also reflected on her coaching mistakes:

I blame myself because I kind of knew that I needed to rest her more, and I wish I was stronger in my conviction with that but the athlete said she felt good and she's a good kid, she would never lie or anything. So, I wish I handled that differently.

Emily (women's rowing) similarly explained:

As a coach, you're going to make mistakes. And that's where, as a coach, you also want to have a support system around you, so that when you blow it, because you are, because you're a human, that someone else is there to say, "No, no, no, she didn't mean that. She really does care, and she wants you to move forward."

Tanya (women's volleyball) voiced her opinion on mistakes regarding treating injured athletes fairly:

I think there are certain circumstances where the starters are getting more time or pushed harder to come back as opposed to a reserve player. But I don't think that's right. I don't think that that's how you're supposed to handle it.

Mason (football) similarly explained:

They all need to be treated equally as human beings, and not just number 33 . To me, I get mad when people call me "Coach" to be honest with you, because that's what I do for a living. My name's Mason. And when you take the player, and he becomes a player only to you, you're dehumanizing the sport, you're dehumanizing the young man. 
The coaches also reflected considerably on the struggles faced by their injured athletes, including mental, physical, and social pressures. For example, many of the coaches reflected on the emotions experienced by injured athletes, especially when disconnected from their teammates as Owen (men's basketball) described:

Yeah, I would think that's a little bit depressing for some of those guys. It's hard for them to stay engaged because they're sitting over there by themselves for the most part. So, I would think that's pretty depressing and kind of sad for those guys.

Male coaches of male teams specifically discussed athletes' concern over being perceived as weak while injured. Baseball coach, Howard, explained:

There's an age-old fear in athletics of a player, any player, that's, "Are they sore or are they injured?" And the coach wants you to fight through the soreness, the trainer wants you to get off the field in the injury. There's a fine line there. We have to gauge what is what, and the player's always going to say he can fight through, because they never want to be accused of being soft or scared.

Intrapersonal knowledge on personal athletic experiences. All the coaches interviewed spoke to how their personal experiences as an athlete impacted their approach to coaching their injured athletes. Coaches described the ways they experienced injury as an athlete and used these reflections to inform their current practices. Ten of the coaches had experienced injuries themselves as college athletes and tried to focus on how they felt during those times, such as Allison (men's and women's swimming and diving):

Other than knowing that I went through it, I know what it feels like to be injured and the first time I got injured, I kind of allowed...I kind of settled. It was easier to just settle and not really deal with it each time it got better. Second time when I got injured, I realized 
that I was making excuses before and I don't want to make excuses, I want to get over it and figure out how to move forward. So, I've kind of been in both of those shoes, so I think the mindset, you can kind of realize where the most student athletes are.

Wyatt (women's ice hockey) described the experience of being left behind when he was injured: I was hurt, so I went through it. I missed four games in my career so I know what it was like. And two of those games, they went on a long trip. The team went away and I was left behind and it was tough.

David (women's soccer) addressed the difficulties he faced when going to practice while injured, "I know it's frustrating because I went through it myself, where you're at practices. Everybody else is practicing. You feel like you're not contributing to the team in any way, shape or form." Noah (men's and women's tennis) echoed the reflections of other coaches' experiences and extended this to describe the importance of remembering what it felt like to be an athlete:

One thing that I hope I never lose, and don't think I've lost, is what it feels like to be a player. And sometimes I see other coaches where, when they're yelling at their players and getting in their face for choking, or missing shots, or whatever, or losing a match, I'm like, "Gosh! It's like this person was never a player. Like you never had a bad match, you never messed up?" How could they act or pretend like they're so perfect as a coach or they never screwed up as a player?...I do put myself in their shoes, I try to keep an athlete's perspective as I'm approaching situations knowing that, "Hey, it's not fun. The last thing that this kid wanted to do is get injured, and have to sit out practice or matches." 
Some of the coaches reflected on changes to the recovery process since they were college athletes. The coaches reflected not only how medicine and technology have changed, but also how the perceptions surrounding injury has changed as well. Mason (football) explained:

I look at what they have now, and I'm like, "Wow." From everything they do. From how they prepare, to how they rehabilitate, to the care that they get, and the things that are done with them, I think is way different. And I do think to a degree, I would say, players accept injury better today than we did back in the day. Injury wasn't accepted well.

Coaches seemed to appreciate the amount of resources available to their athletes that were not a part of their college athletic experiences. Kevin (women's basketball) acknowledged not only are there more resources, but also the importance of the mental aspect of performance has changed:

I will say this, there's a lot more mental part of this now than it was when I played. We didn't have sport psychologists, we didn't have weight coaches that helped us try to develop ourselves better so we wouldn't get injured, we had trainers but we didn't have the doctors' availability and all the stuff that we have now.

\section{An Extreme Case}

Due to the maximum variation sampling strategy used, the author expected there would be divergence in the patterns that would emerge from respondents (Miles et al., 2014). Within the sample of 13 coaches, only one coach appeared to be an extreme case. Miles et al. (2014) explain that cases are categorized as extreme "where there should have been consensus but there wasn't” (p. 303). Although there was consensus with the rest of the sample regarding the importance of communicating with injured athletes, it did not appear that Joe (women's golf) engaged in reflective practice (i.e., intrapersonal knowledge) to improve the quality of his interactions. Joe's interview lasted a total of 10 minutes and most of the questions prompting him 
to reflect on his experiences as an athlete or a coach resulted in a lack of reflection in his response. For example, when asked how his experiences as an athlete influenced his current coaching practices, he responded "I would say, probably nothing at all." When asked about how he would engage with athletes who may experience psychological distress when attending practice while injured, he responded, "I wouldn't really have that problem.” Joe's responses were mostly misaligned with the sample.

\section{Discussion}

The purpose of this qualitative study was to explore collegiate coaches' knowledge related to the provision of social support to injured athletes throughout the phases of rehabilitation and return to competition using the International Sport Coaching Framework (ISCF) as a guiding framework. This section includes a discussion of the major findings as they relate to coaches' roles in providing support during the injury experience, their use of the ISCF knowledge types while coaching injured athletes, and the barriers to fully supporting their injured athletes. Limitations and recommendations for future research are shared.

\section{Coaches' Roles During Athletes' Injury Experience}

College coaches have many responsibilities related to their role as a head coach. When coaching injured athletes, coaches may feel conflicted about how much they should or should not focus on their injured athletes. Ruddock-Hudson et al. (2014) found athletes in their study had little communication with their coaches during their rehabilitation and most of their coaches' energy seemed to be focused on their non-injured teammates. In the current study, coaches cited the importance of communication with both their injured and non-injured athletes. The coaches reported it was important to develop and maintain personal relationships with all their players, and thus established trust that may facilitate the recovery experience. Some coaches believed 
their roles did not change when their athletes became injured, and all coaches agreed they should not lessen their communication with these athletes simply because of their injuries. It is important for coaching educators to emphasize the value of building and maintaining relationships with athletes to improve not only the coach-athlete relationship, but also to facilitate coaches' communication during injuries.

Studies on the dynamic between coaches and athletes during injury rehabilitation have portrayed coaches as neglectful and focused more on winning than athletes' well-being. For example, in Podlog and Eklund's (2007b) study, professional coaches of elite athletes reported their primary role was to help their injured athletes return to competition. However, the results of the current study provided a different perspective; coaches in the current study discussed the need to consider their athletes' welfare (i.e., long-term health, social involvement) during the injury rehabilitation process rather than a quick return to play. The coaches provided specific examples for keeping their athletes involved while injured and addressing athletes' rehabilitation in a more holistic way, such as listening and asking questions about how their athletes are feeling. With the extreme case of Joe, although he did not appear to be purposely neglecting his injured athletes' needs, he did not seem to value using reflection or psychological skills to support his athletes. Fernandes et al. (2014) emphasized the need for a holistic approach that incorporated both social (e.g., staying involved) and psychological (e.g., managing expectations) elements, which supports the approaches taken by coaches in the current study.

By taking a holistic approach to recovery, coaches would theoretically be better able to understand each of their athletes' unique needs as they rehabilitate their injuries. The coaches in the current study emphasized the importance of knowing athletes' limits and how far they can push injured athletes during the rehabilitation process. Podlog and Dionigi's (2010) study of 
professional coaches emphasized the need to take an individualized approach to coaching injured athletes and acknowledge the unique difficulties and stressors faced by injured athletes. In the current study, coaches described the importance of not creating more stress for their athletes during an already potentially stressful time, such as being isolated from teammates. Previous research echoes the importance of injured athletes maintaining a connection to their teammates and coaches during rehabilitation (Bianco, 2001; Podlog \& Dionigi, 2010) and was touched upon consistently by coaches in this study as well. To improve this for prospective coaches, coaching education programs can incorporate information on providing multiple types of social support (i.e., emotional, informational, and tangible support) to address the difficulties and stressors faced by injured athletes.

\section{Coaches' Use of the ISCF Knowledge Types to Support Injured Athletes}

There are many ways coaches can demonstrate how they use each ISCF knowledge type - professional, interpersonal and intrapersonal knowledge. As explained by Maurice et al. (2017), there is a dearth of research on these knowledge types in the context of athletic injury. As injury is an inevitable component of sport participation, coaches need to be prepared to support their injured athletes as they will likely coach an injured athlete during their careers. Coaches are in a prime position to support injured athletes and there is a need to address how the ISCF knowledge types are relevant in the context of injury.

Professional knowledge in the context of injury includes providing information about the injury but also providing athletes with resources to facilitate their recovery. An important aspect of professional knowledge is understanding what resources are available and the best ways to utilize them to aid athletes in their recovery (Maurice et al., 2017). The coaches in this study demonstrated knowledge of how to build networks of resources and agreed the ability to use and 
trust resources is an important element of supporting injured athletes. The capacity to use networks of individuals may be inherent to the nature of coaching at the collegiate level as most head coaches have assistant coaches. Podlog and Dionigi (2010) found that elite level coaches coordinated the development and maintenance of support networks for their injured athletes, directing them to experts that assisted injured athletes in their recovery. Most coaches in this study were already familiar with the support personnel available to them (e.g., assistant coaches, medical staff) and did not find it difficult to continue to reach out to others to get help for their athletes. Even within the extreme case, Joe, he identified the importance of communicating with his athletic trainers to support his injured athletes. An additional resource coaches can utilize is psychological skills training. While athletes were actively rehabilitating their injuries, coaches in this study focused on the use of psychological skills, such as visualization and goal setting, much like the coaches studied by Podlog and Eklund (2007b). Most coaches are not Certified Mental Performance Consultants, but they can expand their professional knowledge by learning to implement basic psychological skills with injured athletes, such as goal setting, into their coaching practices. Incorporating elements of psychological skills training into coaching education can greatly impact coaches' abilities to help athletes during their injury rehabilitation by expanding their professional knowledge.

Another component of professional knowledge to consider in the context of athletic injury is the use of instructional strategies to adapt training and workouts for injured athletes. Coaches could easily leave injured athletes on the sidelines until they have been cleared to return; however, as the coaches in the current study demonstrated, finding ways to integrate these athletes into practice and training helped ameliorate the effects of being isolated from their team while they recovered. In their study on coaching effectiveness in the context of non-injured 
athletes, Mohd Kassim and Boardley (2018) described the importance of coaches providing athletes the opportunity to master "technical, tactical and physical aspects of sport" as a means of improving athlete outcomes (p. 195). The coaches in the current study appeared to understand that, when possible, injured athletes are still capable of mastering technical, tactical, and physical elements of their sport if coaches have the professional knowledge to tailor training plans. Further, all the coaches understood there was a period of re-integration that would occur to allow the athletes to efficiently and effectively return to competition both mentally and physically. To assist coaches in modifying training plans and transitioning athletes back into participation, coaching education programs can incorporate the use of hierarchal decision-making models (Abraham, Collins, \& Martindale, 2006) to assist coaches in problem solving and evaluating consequences.

The best way for coaches to understand injured athletes needs as they try to involve them in training and practice is to communicate and interact with athletes on a frequent basis. One of the fundamental coaching skills is demonstrating interpersonal knowledge through communicating and interacting with others (Bowes \& Jones, 2006). Coaches stressed the importance of maintaining contact with injured athletes throughout the entirety of their rehabilitation. During these conversations, coaches recommended asking and validating injured athletes in their feelings. Research indicates, in some cases, athletes do not want to talk about emotions and can hide the physical pain they are experiencing (Baugh, Meehan, Kroshus, McGuire, \& Hatfield, 2019; Mayer \& Thiel, 2018). Some of the male coaches explained their male athletes were worried about perceptions of weakness when admitting they were injured. Previous research has found that athletes who play through injury are often regarded as heroic and tough while those who admit to their injuries are mocked (Ginis \& Leary, 2004; Jessiman- 
Perreault \& Godley, 2016). One way to address this concern is to reject the culture of risk, which encourages athletes to jeopardize their health and play through their injuries to win at all costs and retain their starting position, and instead create a culture that supports well-being (JessimanPerreault \& Godley, 2016; Nixon, 1993). The ability for coaches to create this culture, however, will be difficult as they and their athletes have been socialized by ideals of the sport ethic. Sport is a critical venue for the socialization of youth and "sport occurs in a cultural context that normalizes and glorifies risk, pain, and injury" (Young, 2012, p. 102). As young athletes mature to become college athletes, their view on their sport as a culture of risk has been internalized and they are more likely to overconform to the sport ethic (Hughes \& Coakley, 1991; JessimanPerreault \& Godley, 2016). Jessiman-Perreault and Godley (2016) reported 70\% of their sample of college athletes played while injured; more specifically, $23.2 \%$ of females and $15.8 \%$ of males played through injury, suggesting that although men in their study were more likely to overconform to the sport ethic, women were more likely to play through pain and injury. The experiences of some of the male coaches in this study reflected this finding.

In contrast to professional and interpersonal knowledge, demonstrating intrapersonal knowledge is more difficult to detect as it primarily consists of reflections and critiques of self and others. The reflections contain an evaluative piece as well that is meant to improve their coaching practices, whether this is connected to their pedagogy or interactions with athletes (Maurice et al., 2017; Côté \& Gilbert, 2009). The coaches in the current study were prompted to reflect on their experiences as athletes and coaches and how these experiences informed their current coaching practices. The coaches in turn reflected on what they perceived to be quality interactions with their injured athletes and how to continuously improve those interactions, such as keeping only relevant people involved (e.g., medical staff) and asking athletes for feedback 
(e.g., "How can I improve?"). This process of questioning and evaluating their practices and values as a coach is known as critical reflection and is considered a crucial process for coaches to be learn and improve (Cushion, Ford, \& Williams, 2012; Ghaye, 2001). Gallimore, Gilbert and Nater (2014) found incorporating feedback from athletes with elements of professional knowledge (i.e., theory and pedagogy) helped coaches improve the outcomes for their athletes and continue improving as coaches. Several of the coaches in the current study cited examples of when they handled cases of injury poorly and felt the repercussions in their relationships with those athletes. The extreme case, Joe, was the only coach who did not demonstrate an eagerness to reflect on or seek out feedback regarding his interactions with athletes. Coaches who effectively demonstrate intrapersonal knowledge can critique those interactions and find ways to improve these in the future.

The coaches were also asked directly about their personal experiences with injury and how this impacted their current approach to coaching injured athletes. The coaches found reflecting on their experiences as an injured athlete (Cushion, 2011) helped them to keep an open mind about their athletes' injuries. As the coaches reflected on their injuries and the impact those experiences had on their lives, the coaches appeared to understand the long-term effects of the decisions they make about their injured athletes. Specifically, all the coaches emphasized the need to reflect on the big picture because long-term health and well-being is more important than a win-loss record. As with changing the culture of the team, changing the mentality of the importance of winning in college sports will be difficult. Coaches' positions as head coaches are frequently assessed based on their ability to win (Frey, 2007), leading some coaches to focus on those who are physically able to perform (Vergeer \& Hogg, 1999). If coaches find themselves feeling pressured to focus on winning, it may be necessary to reflect on ways they can better 
support their athletes as individuals. The athletes' emotional responses were an important part of how the coaches reflected on their athletes' injury experiences as well. Several of the coaches had coached both men and women in their respective sports and noted the gender of their athletes sometimes impacts how they approach emotional responses. As coaches engage in reflection, they should prompt themselves to consider how societal gender norms and stereotypes may be impacting how they engage with their injured athletes. If coaches are being biased by the gender of their athletes, this may impact how coaches interpret the severity of injuries and lead to differential treatment of athletes (Malcom, 2006; Nixon, 1996). When athletes' pain and injuries are not taken seriously because of gender biases, this has negative consequences for their injury rehabilitation and coach-athlete relationship (Nixon, 1996). The coaches acknowledged it is important to share with athletes that they are aware of what their athletes are experiencing and do so without judgment.

\section{Coaches' Perceived Barriers to Providing Support to Injured Athletes}

This study aimed to gain the perspective from head coaches regarding the barriers they face when trying to provide social support, which were woven throughout coaches' demonstration of professional, interpersonal, and intrapersonal knowledge. The results of the current study suggested coaches wanted to support their injured athletes and believed their coaching peers wanted to do the same. The coaches specifically described barriers related to their ability to communicate effectively with their injured athletes. Christakou and Lavallee (2009) found effective communication between injured athletes and their recovery support team had the potential to facilitate rehabilitation outcomes. However, coaches in the current study cited the athletes' unrealistic expectations for recovery and dishonesty surrounding the injury. If athletes 
are not being honest with coaches about their injuries or maintaining unrealistic expectations for their recovery, the coaches may struggle to communicate and support them.

Another barrier some coaches discussed in the current study was having access to resources at their university that provide support for injured athletes. Within NCAA DI programs, there can be significant variations in the types of resources available to athletes and coaches at these schools. Smaller DI programs, especially those outside the power five conferences, have fewer resources to begin (Won \& Chelladurai, 2016) and according to the coaches in this study, face difficulties accessing them. The differences cited by the coaches, such as access to the training room, may seem inconsequential to the overall success of the program, but impacts the resources available to support athletes.

However, coaches are still capable of supporting their athletes despite their limited access to resources. Social support, in some instances, is as simple as sending a text message to check in with athletes after a doctor's appointment or asking how they are feeling that day. If coaches are unsure of how to support their athletes, merely asking the athlete how the coaching staff can be of assistance to the athlete can be impactful. When coaches are taught ways to provide support that are simple, this may allow coaches to provide support in greater quantities and better quality to their athletes. To assist coaches in improving the support offered, it is valuable to consider how coaches developed their support behaviors. Many of the coaches in this study, and likely across the NCAA, have framed their coaching practices based on their experiences when they were athletes (Cushion, 2011); by doing this, it appears that coaches may be maintaining the status quo which could impact their motivation to improve support for their athletes (e.g., reflecting on improving communication within the support network) in the present. Cushion (2016) challenged the discourse surrounding the reflective nature of coaching (the use of 
intrapersonal knowledge), stating that "reflection is implemented in coaching to accommodate existing culture rather than change it" (p. 91). Perhaps coaches can be challenged on this notion of maintaining the status quo and push to create an environment that is beneficial to the athletes currently experiencing it. Coaching education programs may be a critical step in changing the status quo and future generations of coaches through varied learning approaches that challenge the traditional discourse.

\section{Limitations}

This study was limited to the perspectives of 13 NCAA DI coaches across all nine regions of the United States. Given the purposive sampling method employed, it is possible the coaches who volunteered for the study were those who were most interested and reflective on the topic of social support and injured athletes. Thus, coaches who are not interested in how social support impacts their athletes or have little desire to discuss their approach to injury may not have been reached or chose not to participate. In addition to possible volunteer bias, the coaches' responses may have been influenced by recall bias. The coaches were not asked about every instance of injury they have dealt with and may have recalled the experiences of athletes who they perceived had simple recoveries and with whom they have maintained a positive relationship. To address this in the future, more specific inclusion criteria may be necessary to focus on coaches whose experiences include severe injuries or complicated recovery. Further, although the researcher could not eliminate socially desirable responses from coaches, she emphasized the value of each coaches' voice and experiences rather than leading coaches to respond in a prescribed pattern. Specifically, the researcher reminded coaches of her interest in learning about their unique experiences as the voice of coaches in this context is largely missing. Additionally, the researcher reassured coaches that the information reported would remain 
confidential and there would be no indication of their identities in any report of the results. Some coaches may have been concerned about negative information getting to athletes, their coaching staff, athletic departments, or potentially the NCAA. However, coaches were made aware their responses would remain confidential and there would be no way to connect their identity to the responses they provided to promote honesty throughout the interviews.

\section{Practical Implications}

The athletic injury experience cannot improve without practical ways for coaches to have a positive impact throughout rehabilitation. When considering the ISCF knowledge types as a guide, many coaches already possess the necessary skills to improve their athletes' experiences but may need more direction on implementing this knowledge. First, coaches can use their professional knowledge to help educate their athletes about their injury. Coaches are not medical professionals, nor are they expected to have extensive medical knowledge to provide their athletes. However, much like the coaches in the current study, coaches can offer their athletes informational support by providing them with resources and referrals to individuals who are better equipped to answer questions specific to their injuries. By providing athletes with these resources (e.g., sport psychology consultant, athletes who have experienced similar injuries), coaches are demonstrating an understanding of what their athletes' needs are during rehabilitation and providing them a more informed recovery experience (Gilbert, Lyon \& Wahl, 2015). Coaches can also create a recovery support team for their athletes that includes all the stakeholders in the rehabilitation process, which can also provide athletes with a greater opportunity to have their social support needs met. As supported by the results of the current study, providing athletes with a support team not only creates an environment of caring, but also ensures everyone in the support team is receiving the same information about the process. 
Coaches may already be using professional knowledge to support their injured athletes by using informational social support strategies, such as goal setting and task challenges. Goal setting should include goals beyond their athletics (i.e., academics, personal) to help athletes broaden their identity outside of sport (Dijkstra, Pollock, Chakraverty, \& Ardern, 2017) and should include a timeline for completing the goals as well as time for athletes to engage in selfcare activities. Dijkstra et al. (2017) explained that when athletes expanded their identity beyond athletics, they saw positive outcomes related to both motivation and mental health during rehabilitation. When coaches engage with their athletes in goal setting in aspects of their lives other than sport, coaches have an opportunity to learn more about their athletes beyond the sport context. Some coaches described the importance of also providing task challenges - a type of informational support in which coaches not only encourage injured athletes to engage in goalsetting, but also provide them with information on physical training that will not aggravate their injuries (Gilbert et al., 2015). Providing this type of informational support allows injured athletes to remain connected to their sport and their team as well as develop confidence in their ability to perform (Podlog, Dimmock, \& Miller, 2011).

Injury has a different meaning for everyone, and it is important for coaches to have conversations with their athletes about their current injuries, as well as previous injuries, and what that means to the athletes emotionally. A vital element of emotional support requires that coaches listen without judgment and demonstrate, through their actions, that they are interested and engaged in the social support process (Gilbert et al., 2015). The results of the current study suggested communication is an essential element of interpersonal knowledge for coaches when providing social support. Coaches should ask for athletes' feedback on what has and has not been helpful in their recovery. The athletes' injury rehabilitation should feature the athletes' 
perceptions about the experience to ensure the best outcomes. Allowing athletes to express their needs and frustrations regarding rehabilitation provides coaches with the opportunity to offer emotional, informational, and tangible support to their athletes (Gilbert et al., 2015). In the context of the team itself, coaches hold power when it comes to creating the culture of their teams. If coaches develop a culture of transparency within their team, their athletes will perceive the team as an environment where they are comfortable speaking with their coaches about pain and injury without fear of being mocked or judged (King, Roberts, Hard, \& Ardern, 2018). A culture of transparency is just as important when athletes are engaged in their rehabilitation and preparing to return to play. Injured athletes will be able to better communicate about their progress during rehabilitation but also address their readiness to return to play. By incorporating methods of communicating a new culture which offers support rather than criticism, injured athletes will benefit not only during their rehabilitation but will take those supportive encounters with them into their future endeavors.

Coaches can take the first steps to improving the outcomes for their injured athletes by reflecting on the culture that currently exists in their teams. Using this element of intrapersonal knowledge, coaches should spend time reflecting on improvements they can make to their approach to injury (Stoszkowski \& Collins, 2016). These reflections can be prompted by the coaches themselves or from athletes' feedback. By encouraging an open dialogue about what has been effective for the injured athletes, coaches are offering emotional support to their athletes and gaining valuable insight into how their behaviors impact their athletes. Coaches in situations like those in the present investigation should engage in continuous reflection after each interaction to evaluate what went well, what can be improved and how it can be improved. 
It is worthwhile to also consider the ways coaching education curriculums address demonstrating effective coaching knowledge related to the psychological aspects of injury rehabilitation for those training to become coaches. The International Council on Coaching Excellence (ICCE) uses the ISCF to highlight the knowledge effective coaches should possess in their unique coaching context. Within the ICCE Standards for Higher Education (2016), the organization addresses specific elements of professional, interpersonal, and intrapersonal knowledge requiring interdisciplinary theoretical knowledge. For example, to develop professional knowledge, coaching education curriculums are expected to help coaches in training understand: the process and practice of coaching (e.g., critical thinking and decision making), the context in which they are coaching (e.g., policy development and politics), the sport and sport curriculum (e.g., motor control, philosophy of sport, and sport psychology), and the participant (e.g., anatomy, physiology, and sport psychology) (ICCE, 2016). The development of interpersonal knowledge in coaching education curriculums should be focusing on understanding human relationships (e.g., social learning theory and power dynamics) and pedagogy (e.g., information processing theory) (ICCE, 2016). Intrapersonal knowledge development within the curriculums should focus on understanding the self (e.g., mental skills, epistemology, and interpretations of coaching) (ICCE, 2016). Although the ICCE has set standards for what coaches should be learning, it is valuable to also examine the ways the information within the curriculum is being delivered to coaches in training. Coaches have reported enjoying and learning more from informal and self-directed learning opportunities to interact with other coaches (Stoszkowski \& Collins, 2016). Thus, coaching education programs may consider incorporating their trainees preferred learning methods within their content delivery to improve learning outcomes for future coaches. 


\section{Future Research}

Based on the low response rate from coaches during recruitment, future research should find ways to access the coaches who are struggling to effectively support their injured athletes. Within this sample, Joe may have been a representation of such coaches. Although Joe was analyzed separately as an extreme case, Joe's responses support the need to interview more coaches like him who do not appear to demonstrate the three ISCF knowledge types when working with injured athletes. Gathering information from a sample of coaches like Joe would provide both researchers and coaching educators with valuable information to improve coaches' effectiveness and improve athlete outcomes. Although finding these coaches may be a difficult task, researchers could conduct needs assessments of athletes' social support to identify potential coaches to include in social support intervention studies. Future observational studies should use methods that allow researchers to get a better understanding of how the ISCF knowledge types are demonstrated in real time and gain insight into elements of the context surrounding injuries (e.g., time in the season, athletes' role and status on team) impacting coaches provision of social support. In this type of study, the introduction of a quantitative method to track coaching behaviors, such as the Coaching Behavior Assessment System (CBAS; Smith, Smoll, \& Hunt, 1977) or the Coaching Analysis and Intervention System (CAIS; Cushion, Harvey, Muir \& Nelson, 2012), could be beneficial to providing insight into both the frequency and quality of support behaviors provided. Both the CBAS and CAIS are commonly used instruments in coaching research; however, previous research has not used it to focus directly on the context of athletic injury. With the information provided by the CBAS and CAIS about social support behaviors and the results of studies such as this one, researchers can work towards creating a quantitative measure of coaches' uses of the ISCF knowledge types. 
Additionally, the use of case study designs to study coach-athlete dyads would provide more depth into the discrepancy reported by athletes regarding the support they desire and what is received. To get a better perspective on why this discrepancy exists is to engage both parties in a dialogue about the athlete's rehabilitation and the coach's provision of social support. Athletes have consistently stated their head coaches are not a positive factor during their rehabilitation and the coaches in this study cited athletes as being barriers to providing effective social support. With both members of the coach-athlete dyad citing each other as impediments to a successful recovery, it is crucial that researchers have an opportunity to address this discrepancy within the dyad to extend this to a broader audience. Although the purpose of case studies and qualitative research is not generalization, understanding why the discrepancies exist and what is preventing the dyad from working together is a valuable first step to finding a solution.

Although focusing on the impact of coaches' and athletes' gender was not a primary aim of this study, several of the coaches did bring up gender during their interviews and how it impacted their interpretation of injury severity and response to injury. Bruns (2015) found that injured male and female athletes differed significantly in the amount of emotional support expected and received from coaches before and after an injury. At the onset of injury, female athletes received more emotional support than male athletes, but after the injury, male athletes received significantly more emotional support while female athletes saw significant decreases. Additionally, female athletes had higher expectations for emotional support than male athletes at the onset of injury. Bruns' findings support the need for coaches to make greater considerations about gender when supporting injured athletes. Tomlinson and Yorganci (1997) point to the larger systemic issue of gender power differentials when considering the complexities of the coach-athlete relationship, and the injury experience is not exempt from this issue. Future 
research is necessary to further examine the specific social support needs of injured male and female athletes.

Finally, expanding the level of coaches included in future studies is important to consider as well. Within the collegiate coaching ranks alone, differences may exist between the experiences of Division I, II, and III head coaches based on the nature of expectations and competition at each division. Noting commonalities and differences that may exist across different levels of competition, even within the levels of collegiate sport, can provide insight into the types of experiences faced by most coaches and how to best prepare them to handle these various experiences.

\section{Conclusion}

The purpose of this study was to explore NCAA D1 head coaches' knowledge related to the provision of social support to injured athletes. The ISCF was used as a guiding framework to examine how coaches perceived their role in providing social support throughout the injury recovery process. Additionally, the current study explored the ways coaches used the ISCF knowledge types in the context of supporting their injured athletes. Lastly, this study sought to explore coaches' perceived barriers in providing social support.

The NCAA DI head coaches sampled in this study demonstrated many elements of the ISCF knowledge types within their roles as coaches. Results of the present study suggested a need for coaches to communicate and stay involved with their athletes, whether they are injured and spending their time in the training room or a non-injured member of the team who is engaging in practice and competitions. Communication with athletes, while based primarily as a demonstration of interpersonal knowledge, can aid in the demonstration of other knowledge types as well. All the coaches in the study supported using communication to improve 
interactions with all athletes on their team and their network of professionals. When coaches are asking questions and being empathetic, this provides them with more information and the opportunity to evaluate the choices they made in their interactions with injured athletes. It is important to remember when athletes are frustrated with the support available from their coaches this is not always the fault of the coaches. In some cases, coaches feel restricted by policies existing at the university level and up through the NCAA. Although the coaches in this sample were quite adept at communicating with their athletes, coaches can be more transparent with their athletes about some of the barriers they face to help their athletes understand that while the coaches are trying to support their athletes, there can be times when they are unable to offer support. If the process of supporting injured athletes is to improve, it is vital coaches express how policies in place restrict their abilities to support athletes rather than blaming coaches for not being the perfect examples of social support. 


\section{References}

Abgarov, A., Jeffery-Tosoni, S., Baker, J., \& Fraser-Thomas, J. (2012). Understanding social support throughout the injury process among interuniversity swimmers. Journal of Intercollegiate Sport, 5, 213-229.

Abraham, A., Collins, D., \& Martindale, R. (2006). The coaching schematic: Validation through expert coach consensus. Journal of Sports Sciences, 24(6), 549-564.

Anfara, V. A., Brown, K. M., \& Mangione, T. L. (2002). Qualitative analysis on stage: Making the research process more public. Educational Researcher, 31(7), 28-38. doi: 10.3102/0013189X031007028

Arvinen-Barrow, M., \& Walker, N. (2013). Introduction to the psychology of sport injuries. In M. Arvinen-Barrow \& N. Walker (Eds.), The psychology of sport injury and rehabilitation (pp. 2-5). New York, NY: Routledge.

Baugh, C. M., Meehan, W. P., Kroshus, E., McGuire, T. G., \& Hatfield, L. A. (2019). College football players less likely to report concussions and other injuries with increased injury accumulation. Journal of Neurotrauma. Advance online publication.

Bellamy, K., Ostini, R., Marini, N., \& Kairuz, T. (2016). Seeking to understand: Using generic qualitative research to explore access to medicines and pharmacy services among resettled refugees. International Journal of Clinical Pharmacy, 38, 671-675.

Beron, K. J., \& Piquero, A. R. (2016). Studying the determinants of student-athlete grade point average: The roles of identity, context, and academic interests. Social Science Quarterly, 97(2), 142-160.

Bianco, T., Malo, S., \& Orlick, T. (1999). Sport injury and illness: Elite skiers describe their experiences. Research Quarterly for Exercise and Sport, 70, 157-169. 
24 Bianco, T. (2001). Social support and recovery from sport injury: Elite skiers share their experiences. Research Quarterly for Exercise and Sport, 72(4), 376-388.

Bianco, T. (2007). Sport injury and the need for coach support. In D. Pargman (Ed.), Psychological bases of sport injury (pp. 237-266). Morgantown, WV: Fitness Information Technology.

Bowes, I., \& Jones, R. (2006). Working at the edge of chaos: Understanding coaching as a complex interpersonal system. The Sport Psychologist, 20, 235-245.

Brewer, B. (1993). Self-identity and specific vulnerability to depressed mood. Journal of Personality, 61, 343-364.

Brown, C. (2005). Injuries: The psychology of recovery and rehab. In S. Murphy (Ed.), The sport psych handbook (pp.215-235). Champaign, IL: Human Kinetics

Bruns, C. A. (2015). Gender differences in social support during injury rehabilitation. Electronic Theses and Dissertations, 208, 1-118.

Caelli, K., Ray, L., \& Mill, J. (2003). 'Clear as mud': Toward greater clarity in generic qualitative research. International Journal of Qualitative Methods, 2(2), 1-23.

Carson, F., \& Polman, R. (2012). Experiences of professional rugby union players returning to competition following anterior cruciate ligament reconstruction. Physical Therapy in Sport, 13, 35-40.

Carter, A. D., \& Bloom, G. A. (2009). Coaching knowledge and success: Going beyond athletic experiences. Journal of Sport Behavior, 32(4), 419.

Cassidy, T., Jones, R. L., \& Potrac, P. (2009). Understanding sports coaching: The social, cultural and pedagogical foundations of coaching practice (2nd ed.). London, England: Routledge. 
Chalmers, D. J. (2002). Injury prevention in sport: Not yet part of the game? Injury Prevention, 8 , iv22-iv25.

Charmaz, K. (2006). Constructing grounded theory: A practical guide through qualitative analysis. Thousand Oaks, CA: Sage Publications.

Chelladurai, P. (2007). Leadership in sports. In G. Tenenbaum \& R.C. Eklund (Eds.), Handbook of sport psychology (pp. 113-135). New York, NY: John Wiley \& Sons.

Christakou, A. \& Lavallee, D. (2009). Rehabilitation from sports injuries: From theory to practice. Perspectives in Public Health, 129(3), 120-126.

Clement, D., Arvinen-Barrow, M., \& Fetty, T. (2015). Psychosocial responses during different phases of sport-injury rehabilitation. A qualitative study. Journal of Athletic Training, 50(1), 95-104.

Clement, D. \& Shannon, V. (2011). Injured athletes' perceptions about social support. Journal of Sport Rehabilitation, 20, 457-470.

Collinson, V. (1996). Becoming an exemplary teacher: Integrating professional, interpersonal, and intrapersonal knowledge. Paper prepared for the JUSTEC Annual Conference.

Cooper, S. \& Endacott, R. (2007). Generic qualitative research: A design for qualitative research in emergency care? Emergency Medicine Journal, 24, 816-819.

Corbillon, F., Crossman, J., \& Jamieson, J. (2008). Injured athletes' perceptions of the social support provided by their coaches and teammates during rehabilitation. Journal of Sport Behavior, 31(2), 93-107.

Côté, J., Bruner, M., Erickson, K., Strachan, L., \& Fraser-Thomas, J. (2010). Athlete development and coaching. In J. Lyle \& C. Cushion (Eds.) Sports Coaching: Professionalisation and Practice, 63-84. Oxford, U.K.; Elsevier. 
Côté, J., \& Gilbert, W. (2009). An integrative definition of coaching effectiveness and expertise. International Journal of Sport Science and Coaching, 4(3), 307-323.

Crotty, M. (1998). The foundations of social research: Meaning and perspective in the research process. Chicago, IL: Sage.

Cushion, C. J. (2016). Reflection and reflective practice discourses in coaching: A critical analysis. Sport, Education \& Society, 23(1), 82-94.

Cushion, C. J. (2011). Coaches learning and development. In I. Stafford (Ed.), Coaching children in sport (pp. 57-69). London, U.K: Routledge.

Cushion, C., Ford, P. R., \& Williams, A., M. (2012). Coach behaviours and practice structures in youth soccer: Implications for talent development. Journal of Sports Sciences, 30(15), $1631-1641$.

Cutrona, C. E. (1990). Stress and social support - In search of optimal matching. Journal of Social and Clinical Psychology, 9(1), 3-14. doi: 10.1521/jscp.1990.9.1.3

Cutrona, C. E., \& Russell, D. W. (1990). Type of social support and specific stress: Toward a theory of optimal matching. In B.R. Sarason, I.G. Sarason, \& G.R. Pierce (Eds.), Social support: An interactional view (pp. 319-366). New York, NY: Wiley.

Danish, S. J. (1986). Psychological aspects in the care and treatment of athletic injuries. In P. F. Vinger \& E. F. Hoerner (Eds.), Sports injuries: The unthwarted epidemic (2nd ed., pp. 345-353). Boston, MA: John Wright.

Deroche, T., Stephan, Y., Brewer, B. W., \& Le Scanff, C. (2007). Predictors of perceived susceptibility to sports-related injury. Personality and Individual Differences, 43, 22182228. 
Dijkstra, H. P., Pollock, N., Chakraverty, R., \& Ardern, C. L. (2017). Return to play in elite sport: A shared decision-making process. British Journal of Sports Medicine, 51, 419420.

Etikan, I., Musa, S. A., \& Alkassim, R. S. (2016). Comparison of convenience sampling and purposive sampling. American Journal of Theoretical and Applied Statistics, 5(1), 1-4.

Evans, L., \& Hardy, L., (1995). Sport injury and grief responses: A review. Journal of Sport \& Exercise Psychology, 17, 227-245.

Evans, L. \& Hardy, L. (2002). Injury rehabilitation: A qualitative follow-up study. Research Quarterly for Exercise and Sport, 73(3), 320-329.

Fernandes, H. M., Machado Reis, V., Vilaça-Alves, J., Saavedra, F., Aidar, F. J., \& Brustad, R. (2014). Social support and sport injury recovery: An overview of empirical findings and practical implications. Journal of Sport Psychology, 23(2), 445-449.

Fish, S. (1990). How to recognize a poem when you see one. In D. Bartholomae \& A. Petrosky (Eds.), Ways of Reading: An anthology for writers ( $2^{\text {nd }}$ ed., pp. 178-191). Boston, MA: St. Martin's Press.

Ford, I., \& Gordon, S. (1999). Coping with sport injury: Resource loss and the role of social support. Journal of Personality and Interpersonal Loss, 4, 243-256.

Ford, I., Gordon, S., \& Horsley, C. (1993). Providing social support for injured athletes: The perspective of elite coaches. Sports Coach, 4, 12-18.

Frey, M. (2007). College coaches' experiences with stress - "Problem solvers" have problems, too. The Sport Psychologist, 21, 38-57.

Galli, N., \& Vealey, R. S. (2008). “Bouncing back” from adversity: Athletes' experiences of resilience. The Sport Psychologist, 22, 316-335. 
115 Gallimore, R., Gilbert, W., \& Nater, S. (2014). Reflective practice and ongoing learning: A coach's 10-year journey. Reflective Practice, 15(2), 268-288.

Gearity, B. T. (2012). Poor teaching by the coach: A phenomenological description from athletes' experience of poor coaching. Physical Education and Sport Pedagogy, 17(1), 79-96.

Ghaye, T., (2001). Reflective practice. Faster, Higher, Stronger, 10, 9-12.

Gilbert, J. N., Lyon, H., \& Wahl, M. T. (2015). Coping with the stress of athletic injury: How coaches can help. Strategies, 28(4), 33-39.

Gilbert, W. D., \& Côté, J. (2013). Defining coaching effectiveness: A focus on coaches' knowledge. In. P. Potrac, W. Gilbert, \& J. Denison (Eds.), Routledge handbook of sports coaching (pp. 147-159). London, England; Routledge.

Gilbert, W. D., \& Trudel, P. (2005). Learning to coach through experience: Conditions that influence reflection. Physical Educator, 62(1), 32-44.

Ginis, K. A. M. \& Leary, M. R. (2004). Self-presentational processes in health-damaging behavior. Journal of Applied Sport Psychology, 16(1), 59-74.

Gould, D., Udry, E., Bridges, D., \& Beck, L. (1997). Coping with season-ending injuries. The Sport Psychologist, 11, 379-399.

Green, S. L., \& Weinberg, R. S. (2001). Relationships among athletic identity, coping skills, social support, and the psychological impact of injury in recreational participants. Journal of Applied Sport Psychology, 13(1), 40-59.

Hagglund, M., Walden, M., Bahr, R., \& Ekstrand, J. (2005). Methods for epidemiological study of injuries to professional football players: Developing the UEFA model. British Journal of Sports Medicine, 39(6), 340-346. 
Hardy, C. J., Burke, K. L., \& Crace, R. K. (1999). Social support and injury: A framework for support-based interventions with injured athletes. In D. Pargman (Ed.), Psychological bases of sport injuries ( $2^{\text {nd }}$ ed., pp.175-198), Morgantown, WV: Fitness Information Technology.

Hardy, C. J., \& Crace, R. K. (1993). The dimension of social support when dealing with sport injuries. In D. Pargman (Ed.) Psychological bases of sport injuries (pp. 121-144). Morgantown, WV: Fitness Information Technology.

Horn, T. S. (2008). Coaching effectiveness in the sport domain. In T.S. Horn (Ed.), Advances in sport psychology (pp. 239-267). Champaign, IL: Human Kinetics.

Houston, M. N., Cross, K. M., Saliba, S. A., \& Hertel, J. (2014). Injury-related fear in acutely injured interscholastic and intercollegiate athletes. Athletic Training \& Sports Health Care, 6(1), 15-23.

Hughes, R. \& Coakley, J. (1991). Positive deviance among athletes: The implications of overconformity to the sport ethic. Sociology of Sport Journal, 8, 307-325.

Huml, M. R. (2018). A factor structure examination of athletic identity related to NCAA divisional differences. Journal of College Student Development, 59(3), 376-381.

Hutchison, M., Comper, P., Mainwaring, L. M., \& Richards, D. W. (2011). The influence of musculoskeletal injury on cognition: Implications for concussion research. The American Journal of Sports Medicine, 39(11), 2331-2337.

Hutchison, M., Mainwaring, L. M., Comper, P., Richards, D. W., \& Bisschop, S. M. (2009). Differential emotional responses of varsity athletes to concussion and musculoskeletal injuries. Clinical Journal of Sports Medicine, 19(1), 13-19. 
160 International Council of Coaching Excellence [ICCE]. (2016). ICCE's standards for higher

education bachelor coaching degree programmes - Consultation draft. Leeds, UK: ICCE.

International Council of Coaching Excellence [ICCE], Association of Summer Olympic International Federations [ASOIF], \& Leeds Metropolitan University [LMU] (2014). International Coach Developer Framework (Version 1.1). Champaign, IL: Human Kinetics.

Jessiman-Perreault, G. \& Godley, J. (2016). Playing through the pain: A university-based study of sports injury. Advances in Physical Education, 6, 178-194.

Johnson, J. E., Jutte, L. S., \& Bell, R. J. (2012). Using sport psychology consultants, part I: Why is there a need? Athletic Training \& Sports Health Care, 4(1), 5-6.

Johnston, L. H., \& Carroll, D. (1998). The provision of social support to injured athletes: A qualitative analysis. Journal of Sport Rehabilitation, 7, 267-284.

Johnston, L. H. \& Carroll, D. (2000). Coping, social support, and injury: Changes over time and the effects of level of sports involvement. Journal of Sport Rehabilitation, 9, 290-303.

Jowett, S. (2003). When the "honeymoon" is over: A case study of a coach-athlete dyad in crisis. The Sport Psychologist, 17(4), 444-460.

Jowett, S. (2007). Interdependence analysis and the 3+1 C's in the coach-athlete relationship. In S. Jowett \& D. Lavallee (Eds.), Social Psychology in Sport (pp. 15-28). Champaign, IL: Human Kinetics.

Jowett, S., \& Don Carolis, G. (2003). The coach-athlete relationship and perceived satisfaction in team sports. In XIth European Congress of Sport Psychology Proceedings (pp. 83-84). Copenhagen, Denmark: Det Samfundsvidenskabelige Fakultets. 
183 Kerr, Z. Y., Marshall, S. W., Dompier, T. P., Corlette, J., Klossner, D. A., \& Gilchrist, J. (2015). College sports-related injuries-United States, 2009-10 through 2013-14 academic years. Morbidity and Mortality Weekly Report (MMWR), 64(48), 1330-1336.

King, J., Roberts, C., Hard, S., \& Ardern, C. L. (2018). Want to improve return to sport outcomes following injury? Empower, encourage, provide feedback, and be transparent: 4 habits! British Journal of Sports Medicine, 0, 1-2.

LaVoi, N. M. (2007). Expanding the interpersonal dimension: Closeness in the coach-athlete relationship. International Journal of Sports Science \& Coaching, 2(4), 497-512.

Lorimer, R., \& Jowett, S. (2013). Empathic understanding and accuracy in the coach-athlete relationship. In P. Potrac, W. Gilbert, \& J. Denison (Eds.), Routledge handbook of sports coaching (pp. 321-332). London, England; Routledge.

Madrigal, L. \& Gill, D. L. (2014). Psychological responses of Division I female athletes throughout injury recovery: A case study approach. Journal of Clinical Sport Psychology, 8, 276-298.

Malcom, N. L. (2006). "Shaking it off" and "toughing it out": Socialization to pain and injury in girls' softball. Journal of Contemporary Ethnography, 35(5), 495-525.

Malisoux, L., Frisch, A., Urhausen, A., Seil, R., \& Theisen, D. (2013). Injury incidence in a sports school during a 3-year follow-up. Knee Surgery, Sports Traumatology, Arthroscopy, 21, 2895-2900.

Mankad, A. \& Gordon, S. (2010). Psycholinguistic changes in athletes' grief response to injury after written emotional disclosure. Journal of Sport Rehabilitation, 19, 328-342. 
204 Maurice, S., Kuklick, C. R., \& Anderson, M. (2017). Providing social support to injured athletes:

205

206

207

208

209

210 Applications of the international sport coaching framework. International Journal of Physical Education, Sports and Health, 4(5C), 166-175.

Mayer, J. D., \& Salovey, P. (1997). What is emotional intelligence? In P. Salovey \& D.J. Sluyter (Eds.), Emotional development and emotional intelligence: Educational implications (pp. 3-34). New York, NY: Harper Collins.

Mayer, J. \& Thiel, A. (2018). Presenteeism in the elite sports workplace: The willingness to compete hurt among German handball and track and field athletes, 53(1), 49-68.

McDonald, S. A. \& Hardy, C. J. (1990). Affective response patterns of the injured athlete: An exploratory analysis. The Sport Psychologist, 4, 261-274.

Merriam, S. B. (1988). Case study research in education: A qualitative approach. San Francisco, CA: Jossey-Bass, Inc.

Mertens, D. (1998). Research methods in education and psychology: Integrating diversity with quantitative and qualitative approaches. Thousand Oaks, CA: Sage Publications.

Miles, M. B., Huberman, A. M., \& Saldaña, J. (2014). Qualitative data analysis: A methods sourcebook (3 $3^{\text {rd }}$ ed.). Los Angeles, CA: Sage Publications Inc.

Mitchell, I., Evans, L., Rees, T., \& Hardy, L. (2014). Stressors, social support, and tests of the buffering hypothesis: Effects on psychological response of injured athletes. British Journal of Health Psychology, 19, 486-508.

Mohd Kassim, A. F. \& Boardley, I. D. (2018). Athlete perceptions of coaching effectiveness and athlete-level outcomes in team and individual sports: A cross-cultural investigation. The Sport Psychologist, 32, 189-198. 
Morrey, M. A., Stuart, M. J., Smith, A. M., \& Wiese-Bjornstal, D. M. (1999). A longitudinal examination of athletes' emotional and cognitive responses to anterior cruciate ligament injury. Clinical Journal of Sport Medicine, 9, 63-69.

Nixon, H. L. (1996). Explaining pain and injury attitudes and experiences in sport in terms of gender, race, and sports status factors. Journal of Sport and Social Issues, 20(1), 33-44.

Nixon, H. L. (1993). Accepting the risks of pain and injury in sport: Mediated cultural influences on playing hurt. Sociology of Sport Journal, 10, 183-196.

Olympiou, A., Jowett, S., \& Duda, J. L. (2008). The psychological interface between the coachcreated motivational climate and the coach-athlete relationship in team sports. The Sport Psychologist, 22(4), 423-438.

Pearlin, L. I., \& McCall, M. E. (1990). Occupational stress and marital support: A description of microprocesses. In J. Eckenrode \& S. Gore (Eds.), Stress between work and family (pp. 39-60). New York, NY: Plenum.

Percy, W. H., Kostere, K., \& Kostere, S. (2015). Generic qualitative research in psychology. The Qualitative Report, 20(2), 76-85.

Pierce, G. R., Sarason, I. G., \& Sarason, B. R. (1991). Integrating social support perspectives: Working models, personal relationships, and situational factors. In S. Duck (Ed.), Personal relationships and social support (pp. 173-189). Newbury Park, CA: Sage.

Podlog, L., Dimmock, J., \& Miller, J. (2011). A review of return to sport outcomes following injury rehabilitation: Practitioner strategies for enhancing recovery outcomes. Physical Therapy in Sport, 12, 36-42.

Podlog, L., \& Dionigi, R. (2010). Coach strategies for addressing psychosocial challenges during the return to sport from injury. Journal of Sport Sciences, 28(11), 1197-1208. 
Podlog, L., \& Eklund, R. C. (2006). A longitudinal investigation of competitive athletes' return to sport following serious injury. Journal of Applied Sport Psychology, 18, 44-68.

Podlog, L., \& Eklund, R. C. (2007a). Psychosocial considerations of the return to sport following injury. In D. Pargman (Ed.), Psychological bases of sport injuries ( $3^{\text {rd }}$ ed., pp. 109-130). Morgantown, WV: Fitness Information Technology.

Podlog, L., \& Eklund, R. C. (2007b). Professional coaches' perspectives on the return to sport following serious injury. Journal of Applied Sport Psychology, 1, 44-68.

Powell, J. W., \& Dompier, T. P. (2004). Analysis of injury rates and treatment patterns for timeloss and non-time-loss injuries among collegiate student-athletes. Journal of Athletic Training, 39(1), 56-70.

Reike, M., Hammermeister, J., \& Chase, M. (2008). Servant leadership in sport: A new paradigm for effective coach behavior. International Journal of Sports Science and Coaching, 3, 227-239.

Robbins, J. E., \& Rosenfeld, L. B. (2001). Athletes' perceptions of social support by their head coach, assistant coach, and athletic trainer, pre-injury and during rehabilitation. Journal of Sport Behavior, 24(3), 277-297.

Rosenfeld, L. B., Richman, J. M., \& Hardy, C. H. (1989). Examining social support networks among athletes. The Sport Psychologist, 3, 23-33.

Rotella, R. J., \& Heyman, S. R. (1993). Stress, injury, and the psychological rehabilitation of athletes. In J.M. Williams (Ed.), Applied sport psychology: Personal growth to peak performance ( $2^{\text {nd }}$ ed., pp. 338-355). Mountain View, CA: Mayfield. 
Ruddock-Hudson, M., O’Halloran, P., \& Murphy, G. (2012). Exploring psychological reactions to injury in the Australian Football League (AFL). Journal of Applied Sport Psychology, 24, 375-390.

Ruddock-Hudson, M., O’Halloran, P., \& Murphy, G. (2014). The psychological impact of longterm injury on Australian Football League players. Journal of Applied Sport Psychology, 26, 377-394.

Saldaña, J. (2013). The coding manual for qualitative researchers ( $2^{\text {nd }}$ ed.). Thousand Oaks, CA: Sage Publications.

Sanderson, A. R., \& Siegfried, J. J. (2018). The National Collegiate Athletic Association Cartel: Why it exists, how it works, and what it does. Review of Industrial Organization, 52, 185-209.

Sarason, I. G., Sarason, R. S., \& Pierce, G. R. (1994). Social support: Global and relationshipbased levels of analysis. Journal of Social and Personal Relationships, 11, 295-312.

Schwandt, T. A. (1998). Constructivist, interpretivist approaches to human inquiry. In N. K. Denzin \& Y. S. Lincoln (Eds.), The landscape of qualitative research: Theories and issues (pp. 221-259). Thousand Oaks, CA: Sage Publications.

Shumaker, S. A., \& Brownell, A. (1984). Toward a theory of social support: Closing conceptual gaps. Journal of Social Issues, 40, 11-36.

Sloane, P. (2008). Coaching experience, playing experience and coaching tenure: A commentary. International Journal of Sports Science and Coaching, 2, 117-118.

Smith, B., \& McGannon, K. R. (2017). Developing rigor in qualitative research: Problems and opportunities within sport and exercise psychology. International Review of Sport and Exercise Psychology, 1-21. doi: 10.1080/1750984X.2017.1317357 
293 Smith, A. M., Scott, S. G., \& Wiese, D. M. (1990). The psychological effects of sport injuries: Coping. Sports Medicine, 9(6), 352-369.

295 Smith, R. E., Smoll, F. L., \& Hunt, B. (1977). A system for the behavioral assessment of athletic coaches. The Research Quarterly, 48, 401-407 in context and practice: Skills, strategies, and techniques. Hoboken, NJ: John Wiley \& Sons, Inc.

Steadman, J. R. (1993). A physician's approach to the psychology of injury. In J. Heil (Ed.), Psychology of Sport Injury, (pp. 25-32). Champaign, IL: Human Kinetics Publishers.

Stringer, E. T. (1999). Action research (2 $2^{\text {nd }}$ ed.). Thousand Oaks, CA: Sage Publications.

Stoszkowski, J., \& Collins, D. (2016). Sources, topics, and use of knowledge by coaches. Journal of Sport Sciences, 34(9), 794-802.

Taylor, J., \& Taylor, S. (1997). Psychological approaches to sport injury rehabilitation. Gaithersburg, MD: Aspen Publication.

Tomlinson, A., \& Yorganci, I. (1997). Male coach/female athlete relations: Gender and power relations in competitive sport. Journal of Sport \& Social Issues, 21, 134-155. Applied Sport Psychology, 15, 279-293.

311 Tracy, S. J. (2010). Qualitative quality: Eight 'big-tent' criteria for excellent qualitative research. Qualitative Inquiry, 16, 837-851. communicating impact. Malden, M.A.: Wiley-Blackwell. 
315 Udry, E. (1999). The paradox of injuries: Unexpected positive consequences. In D. Pargman (Ed.), Psychological bases of sport injury (pp. 79-88). Morgantown, WV: Fitness Information Technology.

Udry, E., Gould, D., Bridges, D., \& Tuffey, S. (1997). People helping people? Examining the social ties of athletes coping with burnout and injury stress. Journal of Sport \& Exercise Psychology, 19, 368-395.

Vergeer, I. \& Hogg, J. M. (1999). Coaches' decision policies about the participation of injured

Wadey, R., Evans, L., Evans, K., \& Mitchell, I. (2011). Perceived benefits following sport injury: A qualitative examination of their antecedents and underlying mechanisms. Journal of Applied Sport Psychology, 23, 142-158.

Walker, N., Thatcher, J., \& Lavallee, D. (2010). A preliminary development of the Re-Injury Anxiety Scale (RIAI). Physical Therapy in Sport, 11, 23-29.

Wiese-Bjornstal, D. M., Smith, A. M., Shaffer, S. M., \& Morrey, M. A. (1998). An integrated model of response to sport injury: Psychological and sociological dynamics. Journal of Applied Sport Psychology, 10, 46-69.

Williams, J. M. \& Andersen, M. B. (1998). Psychosocial antecedents of sport injury: Review and critique of the stress injury model. Journal of Applied Sport Psychology,10(1), 5-25.

Won, D., \& Chelladurai, P. (2016). Competitive advantage in intercollegiate athletics: The role

335 Yang, J., Peek-Asa, C., Lowe, J. B., Heiden, E., \& Foster, D. T. (2010). Social support patterns of collegiate athletes before and after injury. Journal of Athletic Training, 45(4), 372-379.

337 Young, K. (2012). Sport, violence, and society. London: Routledge. 
Athletic injury is an experience that is common and almost inevitable (Chalmers, 2002). Brown (2005) suggested that "serious athletes come in two varieties: those who have been injured, and those who have not been injured yet" (Arvinen-Barrow \& Walker, 2013, p. 2). For

342 this review and study, injury will be operationalized as "a physical complaint resulting from

343 sports participation that forces the athlete to interrupt or modify his usual training plan for at 344 least one training unit” (Malisoux, Frisch, Urhausen, Seil, \& Theisen, 2013, p. 2896). This

345 definition may also be thought of as a time-loss injury as it depicts a physical complaint that

346 results in loss of participation time in their sport.

\section{Psychological and Emotional Responses to Injury}

When an athlete first sustains an injury, a full range of emotional responses manifest

349 (Bianco, 2007). In some instances, athletes may experience sadness, anger, frustration, or

350 disappointment (Bianco, Malo, \& Orlick, 1999; Tracey, 2003) while in others an athlete may

351 perceive some benefits to being injured (Ford \& Gordon, 1999; Udry, Gould, Bridges, \& Beck,

352 1997; Wadey, Evans, Evans, \& Mitchell, 2011). While most athletes will experience an injury in

353 varying degrees of severity, even those who have sustained similar injuries will not have a

354 similar injury experience. The injury recovery and rehabilitation process can never actually be

355 predetermined due to the unique recovery path and obstacles athletes experience and their

356 cognitive appraisal of the injury experience.

$357 \quad$ For those athletes experiencing a negative psychological and emotional response to their 358 injury, these feelings may include but are not limited to denial, depression, anxiety, grief, anger, 359 and sadness (Johnson, Jutte, \& Bell, 2012; Tracey, 2003). Negative responses to the onset of 360 injury should not be seen as harmful, but rather as a normal response to a traumatic event 
361 (Mankad \& Gordon, 2010; McDonald \& Hardy, 1990). Morrey, Stuart, Smith, and Wiese-

362 Bjornstal (1999) found that athletes who had undergone anterior cruciate ligament (ACL)

363 surgery and had negative initial responses to this injury had minimal negative impact on their

364 physical recovery six months after their surgery. In this study of athletes' psychosocial and

365 physical recovery, these athletes experienced significant mood changes as they progressed

366 through their rehabilitation (Morrey et al., 1999). Their mood mirrored their perception of

367 progress, with improvements in recovery leading to improved moods and difficulties being

368 associated with more mood disturbances (Evans \& Hardy, 2002; Morrey et al., 1999).

369

Grief is a typical response to injury that leads athletes to experience a sense of loss

370 (Evans \& Hardy, 1995; McDonald \& Hardy, 1990). This loss is usually connected to some

371 component of the individual, such as athletic identity (Brewer, 1993; Johnson et al., 2012) or

372 physical capacity (Evans \& Hardy, 2002), and may be experienced as a sudden, gradual, or

373 temporary loss (Mankad \& Gordon, 2010). Athletes who are unable to process their grief,

374 especially those with higher athletic identity (Brewer, 1993; Green \& Weinberg, 2001), may

375 experience a greater number of negative consequences for their rehabilitation and return to

376 competition (Mankad \& Gordon, 2010). If an athlete is required to sit out from competition or

377 practices, this lack of participation may contribute to loss of social connection and belonging

378 (Mankad \& Gordon, 2010) and act as a motivator for the athlete to return as soon as possible

379 (Podlog \& Eklund, 2006). In Ruddock-Hudson, O’Halloran, and Murphy's (2012) research in the

380 psychological reactions to injury in Australian Football League players, a common theme

381 amongst their participants was increased feelings of isolation which led to increased difficulty

382 during rehabilitation and an influx of negative emotions throughout the recovery process. These

383 athletes reported that emotional responses to their injuries, such as depression and devastation, 
384 varied depending on the severity of the injury but also indicated that the longer they were out of

385 participation, the more moody and anxious they became (Ruddock-Hudson et al., 2012). As

386 reflected in Ruddock-Hudson et al. (2012) study, athletes' emotional responses to their injury do

387 not stop once the athlete returns to competition and anxiety regarding rehabilitation and fitness

388 levels remain present throughout their integration back into participation.

As athletes rehabilitate their injuries, there is the potential for re-injury anxiety or the fear

390 of re-injury to develop. There is a debate in the literature about whether this concern of re-injury

391 is fear or anxiety (Walker, Thatcher, \& Lavallee, 2010). As Walker et al. (2010) explain, fear is

392 connected to a certain danger that is present while anxiety represents more of an ambiguous and

393 uncertain potential for the injury to reoccur. Maurice and Clement (in review) found that the

394 difference in whether the athlete experiences fear or anxiety is connected to the type of sport the

395 athlete plays (i.e., contact, collision, or non-contact). Maurice and Clement (in review) put forth

396 the hypothesis that fear of re-injury is more prevalent in collision sports where physical contact

397 is guaranteed on every play, but this hypothesis needs further examination. Athletes can spend

398 months rehabilitating an injury and can have all that hard work taken away in an instant if they

399 do become re-injured upon returning to sport (Podlog \& Eklund, 2006). Podlog and Eklund

400 (2006) found that most their participants feared re-injury, especially those who were injured for

401 the first time and were unsure of how a return from injury would look. The participants in this

402 study explained concerns over inserting themselves into routine situations that caused the injury

403 and an inability to perform at the level that was expected (Podlog \& Eklund, 2006). Carson and

404 Polman (2012) found that rugby players returning from an ACL reconstruction were focusing on

405 regaining confidence and focused on making a successful return, but also very concerned about

406 contact and feared re-injury upon returning to competition. A critical piece of rehabilitating an 
407 injury is feeling confident in the injured area and the rehabilitation process (Carson \& Polman,

408 2012; Taylor \& Taylor, 1997). Carson and Polman (2012) found that athletes felt reassured when

409 members of their treatment provided reassurance that they worked hard to rehabilitate the injury

410 properly, but were not able to feel fully confident until they had the ability to test out the injured

411 area during competition. The participants in the Podlog and Eklund (2006) study reported that

412 their fears were completely absolved upon testing the injury in their return, but most still held on

413 to the fear of re-injury even after they made a successful return.

414 While the negative response to injury is a common and expected outcome of sustaining

415 an injury, many athletes who also perceive some benefit to being injured (Wadey et al., 2011).

416 During their rehabilitation, athletes may begin to increase their mental toughness through this

417 time of adversity and give them a new perspective on their involvement in their sport (Wadey et

418 al., 2011). For some athletes, being able to take a break from their sport involvement and

419 becoming more isolated was a benefit rather than a negative consequence to being injured

420 (Podlog \& Eklund, 2006). Ruddock-Hudson, O’Halloran, and Murphy (2014) found the most

421 common behavioral response during the initial phase for athletes was distancing themselves from

422 the team environment. Just as with the negative emotional responses to injury varying throughout

423 rehabilitation, the perceived benefits will also change as the athlete progresses through recovery

424 and returns to competitions. According to Wadey et al. (2011), in the initial onset of injury, the

425 recognition of a need for support, initiating contact with their social support network, and being

426 more reflective of their emotions were the most commonly reported benefits. While athletes

427 rehabilitated their injuries, common benefits included free time, more individualized and sport-

428 specific training, a focus on academics and the potential to take on the role as a player-coach

429 (Wadey et al., 2011). As athletes returned to competition, they found that their ability to cope 
430 with adversity had improved and reported increased resilience (Galli \& Vealey, 2008; Wadey et

431 al., 2011).

432 Stages of Injury Rehabilitation

Athletes who have sustained an injury are quite likely experiencing stress and/or a

434 negative emotional response (Smith, Scott, \& Wiese, 1990). For some athletes, sustaining an

435 injury is the most difficult thing that will happen to them (Bianco, 2007; Danish, 1986). Injured

436 athletes require support in the three stages of the injury and recovery process: occurrence of

437 injury, treatment and recovery, and return to full competition (Bianco, 2007).

In the occurrence of injury stage, the athlete has just sustained an injury and is likely unaware of the consequences that may follow. During this time, athletes may feel anxious,

440 frustrated, scared, and/or angry about becoming injured (Johnston \& Carroll, 2000; Madrigal \&

441 Gill, 2014; McDonald \& Hardy, 1990). The athlete's perception of the severity of the injury will

442 play a role in how they will cognitively appraise the injury (Bianco, 2007), with more severe

443 perceptions leading to more negative appraisals (Clement, Arvinen-Barrow, \& Fetty, 2015). The

444 way the athlete cognitively appraises the injury will depend on a myriad of personal (e.g., age,

445 history of injury, and coping skills) and environmental factors (e.g., life stress, social support, 446 and timing of the injury; Bianco, 2007).

447 Once the athlete has received a diagnosis, they may begin to seek treatment for the injury.

448 In this stage, the athlete may be required to seek out treatment from a physical therapist and/or

449 other medical professionals to rehabilitate the injury. During this stage, an athlete may begin to

450 face obstacles while working towards goals that were set for rehabilitation. Experiencing

451 adversity or setbacks is not an uncommon occurrence during injury rehabilitation (Rotella \&

452 Heyman, 1993; Steadman, 1993; Taylor \& Taylor, 1997). Not being able to reach the 
453 rehabilitation goals may lead an athlete to feel less motivated and potentially lead to less

454 adherence to the treatment plan (Evans \& Hardy, 2002). An athlete may also begin to question

455 the rehabilitation process and feel frustrated by their physical limitations (Clement et al., 2015).

456 It is important for those working with the athlete to help them maintain motivation to adhere to 457 rehabilitating the injury (Bianco, 2007).

458 When the athlete has completed rehabilitation, and has been cleared to return to 459 competition, they have entered the final stage of the injury and recovery process. In some 460 instances, an athlete appears to be ready to compete physically but is not mentally ready to get 461 back to competition (Podlog \& Eklund, 2007a). In cases, such as these, re-injury anxiety may 462 have an impact on the performance of the individual and increase the likelihood that the athlete 463 will sustain another injury (Taylor \& Taylor, 1997). For others, the rehabilitation experience may 464 have created an appreciation for what the athlete's body can handle and improved the athlete's 465 mental toughness (Clement et al., 2015; Udry, 1999).

466

\section{Injury response model}

The Wiese-Bjornstal, Smith, Shaffer, and Morrey (1998) integrated model of response to sport injury is regarded as one of the most thorough and well-developed cognitive appraisal models of injury (Brewer, 2000). The model examines both pre-injury and post-injury factors as they relate to the individual's response to injury with both physical and psychological recovery being the outcome (Wiese-Bjornstal et al., 1998). The pre-injury factors represented in the Wiese-Bjornstal et al. (1998) integrated model (i.e., personality, history of stressors, coping resources, and interventions) are like those highlighted in the Williams and Andersen (1998) stress-injury model as antecedents to injury (i.e., personality, history of stressors, and coping resources). 
When an athlete sustains an injury, they have been influenced by pre-injury factors as how they will respond to the injury in addition to personal factors that relate to the injury (e.g., type and severity of injury) and individual differences (e.g., gender, age, disordered eating, and pain tolerance) and situational factors relating to their sport (e.g., type, level of competition, and playing status), social setting (e.g., social support, coach influences, and sport ethic), and their environment (e.g., accessibility to rehabilitation; Wiese-Bjornstal et al., 1998). Both the personal and situational factors have an influence on how the athlete cognitively appraises the injury. An injured athlete's cognitive appraisal (i.e., how they perceive the circumstances of the injury) may be influenced by many factors and have an impact on how they perceive their goals are affected now due to the injury, beliefs about their future, explanations for why the injury happened, or the extent that the athlete feels loss at the onset of the injury (Wiese-Bjornstal et al. 1998). As mentioned in the section on emotional responses, athletes' responses to their recovery comes in many forms and often changes throughout the recovery process, and this is due to their cognitive appraisals. The integrated model presents a set of bi-directional arrows that reflect the path in which the cognitive appraisal influences emotional responses (e.g., anger, depression, grief, and positive attitude) and in turn emotional responses influence behavioral responses (e.g., adherence to rehabilitation, malingering, and use of social support), with Wiese-Bjornstal et al. (1998) noting that this path of influence may be reversed as well. For this review and subsequent study, the aspect of the integrated model that is addressed is the social support that falls under situational factors.

\section{Social Support}

Social support is defined as "an exchange of resources between at least two individuals perceived by the provider or the recipient to be intended to enhance the well-being of the 

518 athlete

recipient” (Shumaker \& Brownell, 1984, p. 13). According to Taylor and Taylor (1997), at the most fundamental and basic level, social support allows the injured athlete to feel that they have someone who is there to help them through the process of rehabilitation and returning to competition. The process of recovering from an injury is a demanding journey and having a sense that someone is there for support can be a critical component in an athlete's recovery process. Social support is not a one size fits all resource for injury. There are eight types of social support that group into three overarching types of support (Hardy \& Crace, 1993) that exist to meet the unique needs of those who have sustained an injury. Each athlete who sustains an injury has a distinctive experience that consists of stressors that may be similar or very different to another injured athlete and require a variety of ways they need support.

\section{Types of Social Support}

Emotional. Emotional support consists of listening support, emotional comfort, and emotional challenge. In its most basic form, emotional support refers to providing a sense of comfort and care to the recipient (e.g. injured athlete), being the shoulder to cry on. According to Johnston and Carroll (1998), emotional support appears to the most important during the beginning phases of injury rehabilitation. Johnston and Carroll (1998) found that emotional support was provided by those who had a close relationship with the athlete but were not necessarily experts in the rehabilitation process or the sport. For the emotional support to be effective, it needed to be received from an individual with an existing relationship with the

Informational. Informational support consists of reality confirmation, task appreciation, and task challenge. It is giving advice about the athlete's situation or providing them with information about their injury and rehabilitation process (Bianco, 2007). During the beginning 
522 phase of rehabilitation, most informational support comes from other injured athletes while the

523 primary sources are physiotherapists and coaches as the athletes prepare to return to competition

524 (Johnston \& Carroll, 1998). Other injured athletes can be a great source of information support

525 for athletes who have just recently sustained an injury. Having the opportunity to speak with

526 another athlete who is going through a similar experience can provide a sense of connection that

527 the athlete may be missing since they have been sidelined. Another benefit is that the other

528 injured athletes may be able to help inspire the athlete to keep up with their rehabilitation and

529 show them that it is possible to get better. Informational support may also come from athletic

530 trainers, physical therapists, and physicians.

531 Tangible. Tangible support consists of material assistance and personal assistance. This

532 type of support can be described as providing concrete assistance to the injured athlete (e.g.

533 giving the athlete a ride to a doctor's appointment). The extent of tangible support provided may

534 depend on the severity of the injury. For athletes who are in casts or are otherwise incapacitated,

535 the provision of social support is imperative for daily functioning (Johnston \& Carroll, 1998).

536 This type of support is usually provided by those the athlete lives with or has regular contact

537 with. Unfortunately for those who do not appear to be in pain or to have a serious injury, tangible

538 support is sometimes withheld because the injury is the less visible.

539 Most the research done with injured athletes, social support, and coaches has focused on

540 the types of social support mentioned above, developed by Hardy and Crace (1993). The only

541 types of support being consistently provided by coaches is informational support (Johnston \&

542 Carroll, 2000; Johnston \& Carroll, 1998; Corbillon, Crossman, \& Jamieson, 2008; Rosenfeld,

543 Richman, \& Hardy, 1989), which may include education about the injury, feedback about their

544 return time frame, or their role on the team. Athletes have reported that they feel as though 
545 coaches do not care about their recovery and that coaches remain distant while the injured athlete

546 recovers (Udry, Gould, Bridge, \& Tuffey, 1997). This type of need falls into the category of

547 emotional support, which is usually found from teammates or friends and family members.

548 When studying college athletes, many times the athlete is living far away from family members

549 and their friends consist primarily of their teammates. As the athlete goes through the

550 rehabilitation process, they may frequently find themselves isolated as their teammates spend

551 most of their time at practice, training, and competitions while they are at their physical

552 therapist's office completing rehabilitation exercises. The role of the coach in providing social

553 support becomes a crucial one when we look to see who the athlete has in their social network to

554 provide them with support during this stressful time.

555

556

557

558

559

560

561

562

563 564 2010).

565

566

567

\section{Role of Social Support in Injury Rehabilitation}

It has been well established that social support is a crucial component of the injury rehabilitation process for athletes (Bianco, 2001; Clement \& Shannon, 2011; Ford, Gordon, \& Horsley, 1993; Mitchell, Evans, Rees, \& Hardy, 2014; Robbins \& Rosenfeld, 2001; Rosenfeld, Richman, \& Hardy, 1989; Udry, Gould, Bridge, \& Tuffey, 1997; Yang, Peek-Asa, Lowe, Heiden, \& Foster, 2010). Social support, according to the stress buffering hypothesis, can improve the cognitive appraisals that individuals have about potentially stressful situations, such as injury rehabilitation, and reduce the likelihood of injury and illness (Clement \& Shannon, 2011; Mitchell et al., 2014; Robbins \& Rosenfeld, 2001; Rosenfeld et al., 1989; Yang et al.,

As athletes progress through the stages of their injury recovery and face new challenges, social support becomes a critical element of this process. Clement, Arvinen-Barrow, and Fetty (2015) qualitatively analyzed injured collegiate athlete's psychosocial responses throughout their 
568

569

570

571

572

573

574

575

576

577

578

579

580

581

582

583

584

585

586

587

588

589

590

rehabilitation. The framework for analyzing the results of their interviews was founded in the Wiese-Bjornstal et al. (1998) integrated model of injury response and reports results in the form of cognitive appraisals, emotional responses, and behavioral responses throughout each stage of recovery. In the initial response to the injury, Clement et al. (2015) found that athletes held generally negative appraisals about their injury leading to a more negative emotional response as well. The athletes most frequently reported behavioral response was seeking social support from both teammates and coaches in this first stage of recovery. As athletes received a diagnosis and began to actively rehabilitate their injury, they adjusted their cognitive appraisals of the injury, which in turn had an impact on their emotional response (Clement et al., 2015). During their rehabilitation, the athletes reported the importance of social support from sports medicine professionals and some athletes continued receiving support from their coach. Clement et al. (2015) reported that when athletes returned to competition, their cognitive appraisals were focused on concerns about their rehabilitation, leading them to experience nervousness and anxiety about becoming reinjured. As in the previous two stages, the most common behavioral response again was seeking social support (Clement et al., 2015).

Ruddock-Hudson et al. (2012) suggested that the importance of social support is influenced by the severity of the injury, with more severe, long-term injuries requiring more support throughout the process of recovery. Ruddock-Hudson, O'Halloran, and Murphy (2014) examined the psychological responses to long-term injury with Australian Football League players from the onset of their injury through their return to competition. Ruddock-Hudson et al. (2014) found, like many others, that the initial response to the onset of injury is a negative emotional response. Although the athletes in this study most commonly reported distancing themselves from the team environment, they still sought out social support from their teammates 
591 and family. During this initial stage, athletes reported their coaches having concerns about the

592 injury and reaching out to them following the onset of the injury. As the athletes began to

593 rehabilitate their injury, social support was still sought as they became more isolated from

594 teammates and most athlete reported receiving minimal social support and/or communication

595 with coaches during their rehabilitation (Ruddock-Hudson et al., 2014). The need for social

596 support was still present within their return to sport and the lack of social support from coaches

597 remained an issue for these athletes.

598 While this relationship between injury and social support is well known, the sport

599 psychology literature points out a clear discrepancy between the support an injured athlete needs

600 and what they are receiving, especially from their coaches (Corbillon et al., 2008; Gould et al.,

601 1997; Udry et al., 1997). Coaches can, however, act as a source of support for the athlete's

602 stressors during rehabilitation, yet this is not happening as frequently as athletes are needing. The

603 research on social support in injury rehabilitation focuses primarily on the athlete receiving the

604 social support and little about the coach's provision of social support (Podlog \& Eklund, 2007b).

605

606

607

608

609

610

611

612 al., 2008; Gould et al., 1997; Udry et al., 1997). 
Udry et al. (1997) found that when examining sources of social support for injured athletes, such as coaches, teammates and family members, the most negative influence during the

615 rehabilitation period is coaches. Udry et al. (1997) presented results of injured skiers perceptions

616 about the social support provided by their coaches and found that $67 \%$ of their participants

617 reported coaches having a negative influence during their recovery experience. The themes that

618 fell under negative coach influence were "distant, insensitive to injury, inappropriate/insufficient 619 rehabilitation guidance, lack of belief in athlete, and other" (Udry et al., 1997, p. 383), with 47\% 620 of the athlete reporting coaches being distant. Athletes who reported coaches being insensitive to 621 their injury shared statements expressing that they were "bothersome" or an "afterthought" and 622 that their coaches would not want to speak with them until they completed their rehabilitation 623 programs (Udry et al., 1997, p. 385). The athletes in this study also noted that when their coaches 624 attempted to provide them with support regarding their rehabilitation it was often misguided but 625 “well-intentioned” (Udry et al., 1997, p. 386). Conversely, 52\% of the athletes reported a 626 positive coach influence while recovering from injury through the themes of "stayed emotionally 627 connected, supported and encouraged, and consulted with" (Udry et al., 1997, p. 386). Of these 628 athletes, $52 \%$ reported their coach tried to stay emotionally connected during rehabilitation. The 629 amount of contact and effort put into staying emotionally connected to the athlete ranged from 630 daily visits to several phone calls over a four-month rehabilitation period. For those athletes 631 reporting feeling supported and encouraged, they revealed that they appreciated having a coach 632 who was a source of "emotional support" and someone who "believed in me" (Udry et al., 1997, 633 p. 387$)$.

634 In research that has included coaches as a source of social support for injured athletes, 635 most of the results have shown that there is an incongruence between what the athlete needs and 
636 what they are receiving (Corbillon et al., 2008; Robbins \& Rosenfeld, 2001; Udry et al., 1997).

637 Some coaches have hesitated to provide support to injured athletes to avoid the perceptions that

638 may develop about the relationship with the injured athlete (i.e. playing favorites) or they may

639 believe that the athlete needs to figure it out on their own (Robbins \& Rosenfeld, 2001;

640 Rosenfeld, Richman, \& Hardy, 1989).

641 The presence of coach support can motivate the athletes to work hard through the

642 rehabilitation process and conversely, a lack of support can lower confidence and self-esteem

643 because if you are not playing or contributing, then you do not matter (Bianco, 2001). The

644 perception of support an athlete has will influence how they appraise stressful situations (Bianco,

645 2001; Pierce, Sarason, \& Sarason, 1991; Sarason, Sarason, \& Pierce, 1994). Coach support is

646 highly desired by athletes but research has consistently shown that athletes are seeking and

647 receiving social support from athletic trainers and not their coaches. In some cases, coaches may

648 claim that they were unaware that the athlete needed support, but when examining the coach-

649 athlete relationship, it is not always comfortable or safe to seek support when the coach-athlete

650 relationship is poor. There may also be instances where the athlete seeks out support but the

651 coach does not agree with the degree of distress the athlete presents with and decides to withhold

652 support (Pearlin \& McCall, 1990).

653 Clement and Shannon (2011) studied athletes' perceptions of social support from

654 coaches, athletic trainers, and teammates and found that support from athletic trainers was the

655 most readily available source of support as well as the most useful source. While coach support

656 was rated relatively high in this particular study, research from Hardy, Burke, and Crace (1999)

657 has found that the type of support coaches are most likely to provide is informational support in

658 hopes of getting them back to competition. Clement and Shannon (2011) reinforce that the type 
659 of support being offered needs to match the support needed by the athlete (i.e., optimal matching hypothesis) and suggest that in this sample of athletes may have experienced coaches who did

661 this well when compared to other studies. Additionally, Clement and Shannon (2011) highlight

662 that the athletes in their study were most satisfied with listening support, which falls under the

663 broader theme of emotional support. Athletes who can talk to someone about their emotional

664 response to their injury without concerns about judgmental listening may have a more adaptive

665 rehabilitation experience because they can work through those emotions externally.

666 Some of the incongruences between the injured athlete and the coach may be due to

667 misperceptions from the coach about the type of support the athlete needs. The optimal matching

668 hypothesis of stress and coping is an important component of the stress buffering process and

669 social support (Cutrona, 1990; Cutrona \& Russell, 1990). The optimal matching hypothesis takes

670 into consideration the type of stressor the athlete is experiencing and the corresponding support

671 that is needed for the athlete. For instance, if the athlete's season has ended due to a torn

672 ligament, this is an event that is out of the athlete's control. For stressors that the athlete cannot

673 control, an emotion-based support would be the best match. In some cases, coaches may not have

674 the knowledge about the different types of support and when they are best applied, and athletes

675 may not be aware of what their needs are.

676 There is currently a discrepancy that exists between what injured athletes want from their

677 coaches regarding social support and what they are receiving (Podlog \& Dionigi, 2010).

678 Research on the subject has suggested that coaches are not providing it, but there is also the

679 possibility that coaches do not know how to provide or the different types they can provide.

680 Podlog and Dionigi (2010) interviewed elite level coaches to examine the coaches'

681 strategies for helping athletes through their rehabilitation. The results of this studied suggested 
682 that the coaches in this study were aware of multiple strategies they could use to support their

683 athletes through rehabilitation. The coaches in this study were aware that their approach to

684 helping athletes was not systematic but was dependent upon each athlete's needs. Even when

685 coaches were unable to provide all the support needed by the athlete, the coaches were aware

686 that they could coordinate support through a support network and team approach to

687 rehabilitation. The coaches in Podlog and Dionigi's (2010) study acknowledged that social

688 support was key in their athletes' rehabilitation. The coaches in this study agreed that as the

689 coach they had a role in the injury recovery process but their perspectives varied on what that

690 role was.

691 Coaches have been fired from positions, especially in collegiate and elite levels, for not

692 having a successful season or a history of poor performance from their otherwise talented

693 athletes. When looking at coach success, research has found that the success of the coach is not

694 dependent upon the abilities of their athletes (Sloane, 2008). Coaching success is not defined

695 only by the talent of their athletes. To be an effective coach, one must possess more than just

696 knowledge about the sport or experience playing their sport. Côté and Gilbert (2009) suggest that

697 effective coaches are proficient in more than just sport expertise and use this knowledge to

698 influence athlete outcomes in their specific coaching context. Winning coaches are often

699 considered to be expert pedagogues, as their role is to teach skills and abilities to their athletes

700 (Carter \& Bloom, 2009; Gearity, 2012). Collinson (1996) suggests that a triad of knowledge

701 exists that should be emphasized in the development of exemplary teachers, or in this case,

702 coaches.

703 International Sport Coaching Framework 
The International Council for Coaching Education (ICCE; 2014) has adopted the International Sport Coaching Framework (ISCF) as a resource to aid in the development of coaches' knowledge, development, and certifications (ICCE, 2014). According to Côté and

707 Gilbert (2009), effective coaches should be able to integrate and apply three central types of

708 knowledge: professional knowledge, interpersonal knowledge, and intrapersonal knowledge. The

709 ICCE supports these three central components of coaching effectiveness as competencies that 710 guide coaches' philosophies and values throughout their development.

Professional Knowledge. Professional knowledge is the type of knowledge most

712 commonly thought of when describing coach effectiveness (Côté \& Gilbert, 2009). This type of

713 knowledge relies on sport-specific information and uses declarative and procedural knowledge to

714 teach this information to athletes or assistant coaches (Abraham, Collins, \& Martindale, 2006;

715 Côté \& Gilbert, 2009; Gilbert \& Côté, 2013). Much of this knowledge comes from drawing upon

716 past experiences as an athlete, learning from those who were their coaches (Carter \& Bloom,

717 2009). Having experience as an elite and/or expert athlete has been found to be a common factor

718 among expert coaches. These experiences also played a factor in their development of expert

719 coaching knowledge (Carter \& Bloom, 2009). Once these coaches have become successful, they

720 have often acknowledged that the process of learning more about being a successful coach is

721 ongoing (Carter \& Bloom, 2009). Gilbert and Côté suggest that having a better understanding of

722 a coach's professional knowledge allows researchers to better implement training and

723 educational opportunities to improve coach competencies.

724 One such competency that appears to have a need for improvement is declarative and

725 procedural knowledge on the injury process and how to best help injured athletes. Injury is

726 something that is likely never going to go away in sport and injured athletes are always going to 
727

728

729

730

731

732

733

734

735

736

737

738

739

740

741

742

743

744

745

746

747

748

749

need social support. Previous research has shown that injured athletes are seeking out and receiving the majority of their social support from athletic trainers (Clement \& Shannon, 2011;

Robbins \& Rosenfeld, 2001; Yang et al., 2010), but many still desire social support from coaches but feel that they are not receiving it (Corbillon et al., 2008; Gould, Udry, Bridges, \& Beck, 1997; Udry et al., 1997). Pearlin and McCall (1990) point out that coaches have at times turned away an athlete seeking out support and withheld said support because they do not agree with the degree of distress the athlete presents with. The discrepancies that exist between what athletes and coaches deem appropriate support merit further research to aid in the continuing improvement of athlete outcomes.

Interpersonal Knowledge. Coaches' roles are founded in social interaction. The coach of a team is very rarely acting in isolation and this role is often seen as reciprocally influential in a social context (Collinson, 1996; Côté \& Gilbert, 2009; Gilbert \& Côté, 2013). Interpersonal knowledge, according to Collinson (1996), is what most would refer to as social or people skills. In the coaching position, the aspect of interpersonal knowledge can be overlooked in regards to its importance, especially in influencing athlete outcomes. To be a successful and an effective coach, interpersonal knowledge must include an area of competency that is continually worked on. Côté and Gilbert (2009) recommend that coaches continue to work on developing interpersonal knowledge because coaching success is rarely determined by athletic ability alone. Understanding and communication play a critical role in the development of interpersonal relationships on teams. When considering the injured athlete, it is important for the coach to perceive and attempt to understand the emotions the athlete is experiencing. Emotional intelligence is one way for researchers to better capture how coaches are able to do this. Mayer and Salovey (1997) developed the ability approach to emotional intelligence, which is made up 
750 of four components: identifying, using, understanding, and managing emotions. These four

751 components are critical in understanding how capable a coach is in providing social support to

752 their injured athletes and recognizing when and what is appropriate to give. As mentioned

753 previously, a discrepancy appears to exist that has a negative impact on the support received by

754 the injured athlete. If a coach has the capacity to use interpersonal knowledge, they would be

755 able to recognize the emotions the injured athlete is experiencing and provide the appropriate

756 type of support (i.e. emotional, tangible, informational). Effective coaches are always keeping

757 their athletes' outcomes in mind, and by possessing emotional intelligence and interpersonal

758 knowledge, coaches are putting their best foot forward to aid in the development of their athletes.

759 Intrapersonal Knowledge. Intrapersonal knowledge is often referred to as reflection or

760 insight (Collinson, 1996; Côté \& Gilbert, 2009; Gilbert \& Côté, 2013). Gilbert and Côté (2013)

761 acknowledge that reflection is a central aspect of successful coaching and its value is rarely

762 undermined. While there are several different types of reflections that coaches may engage in,

763 the most important aspect is the role frame, or how the coach is viewing their role. According to

764 Gilbert and Côté (2013), role frames "act as filters through which problems are constructed and

765 strategies are developed" (p. 154). Similar to the experience of observational learning for

766 professional knowledge, the experiences that coaches had as athletes will influence the

767 experiences they have as coaches when it comes to intrapersonal knowledge. The filters that the

768 coach sees the problem through is influenced by past experiences. When reflecting on the

769 behaviors of an injured athlete, or on what to do with an injured athlete, the coach may be

770 influenced by how their coach went about supporting injured athletes. The process of reflection

771 allows the coach the opportunity to examine and reflect on their behaviors to adjust for future

772 success, whether this is with the injured athlete or the style of offense they choose to run (Carter 
773 \& Bloom, 2009; Gilbert \& Trudel, 2005). The ability to have insight and introspect allows

774 coaches to review what is and is not helping their athletes as they keep the athletes' outcomes at

775 the forefront of this reflection.

776 Athlete Outcomes

777 One of the key elements in effective coaching is working towards developing athlete

778 outcomes. According to Horn (2008), effective coaching can be measured by looking at two

779 types of athlete outcomes: successful performance and positive psychological responses. The

780 measurement of performance outcomes is often simpler than the psychological responses, such

781 as motivation, confidence, or perceived competence, and researched far more often (Côté \&

782 Gilbert, 2009). Côté, Bruner, Strachan, Erickson and Fraser-Thomas (2010) have developed a

783 framework from the positive psychology field to conceptualize the athlete outcomes that should

784 emerge in effective coaching interactions. This set of athlete outcomes can be simplified into 4

785 C's: competence, confidence, connection, character/caring.

786 Athlete competence, or performance outcomes as it often seen, has been widely

787 researched and is often looked to the most frequently when examining coach effectiveness

788 because it is the most obvious outcome (Côté \& Gilbert, 2009). A byproduct of this outcome is

789 that coaches are also given the opportunity to influence the remaining three outcomes by helping

790 the athlete develop competence. Previous research has shown that a coach's knowledge and how

791 they behave around the athlete can have a significant impact on the psychological development

792 of their athlete (Chelladurai, 2007; Smith, Smoll, \& Hunt, 1977). These psychological responses

793 could include, but are not limited to, confidence, self-esteem, connection and character (Côté \&

794 Gilbert, 2009). Coaches who act as servant leaders have been found to have athletes who score

795 higher in competence and confidence in sport (Reike, Hammermeister, \& Chase, 2008). Côté and 
796 Gilbert suggest that this study is one of the first to demonstrate the impact of coaches who

797 demonstrate effectiveness with their professional, interpersonal and intrapersonal knowledge.

798 Coaches can create environments where psychological responses can be nurtured and

799 allow for the athlete to become self-determined individuals. Sport is a unique environment that

800 not only allows the participant to develop into a competent athlete but also into a functional

801 member of their society as they learn rules about sportship and fair play as character develops.

802 Jowett (2007) suggested that a factor that influences how well the coach can influence athlete

803 outcomes is the quality of the coach-athlete relationship. Côté \& Gilbert (2009) state that this

804 relationship has a large influence on an athlete's confidence and this relationship is one of the

805 most important factors in the coaching context.

806 Coach-Athlete Relationships

807

Trust and respect are arguably two of the most important components of the relationship

808 between the coach and their athlete (LaVoi, 2007), and this relationship is often the most

809 important relationship that exists in sport (Jowett, 2003). One might argue that trust and respect

810 are critical when the athlete sustains an injury. While there is no causal link between the coach-

811 athlete relationship and subsequent success in sport (Jowett, 2003), the coach-athlete relationship

812 does have some impact on enjoyment and satisfaction in sport.

813 Jowett's (2007) $3+1$ Cs model is a conceptual model that examines the coach-athlete

814 relationship and the interdependence between the two individuals in the relationship. The 3Cs

815 were the initial development in the model (i.e. closeness, commitment, and complementarity)

816 and was later expanded to include the component of co-orientation. Within the construct of co-

817 orientation, direct and meta-perspectives frame how the individual feels about the relationship

818 and their belief in the second individual's feelings about the relationship (e.g. closeness, 
819 commitment, or complementarity). These perspectives are developed and directly linked to the

820 coach-created motivational climate (Olympiou, Jowett, \& Duda, 2005) and have a significant

821 impact on the satisfaction of the athlete in the coach-athlete relationship (Jowett \& Don Carolis,

822 2003). For an athlete who has sustained an injury, an environment that ignores the injured athlete

823 may create confusion and uncertainty for the injured individual. They were once an active part of

824 the team and have now been side-lined. If this athlete believes that there is no longer congruence

825 in the co-orientation aspect of the relationship, this may be potentially due to a lack of empathic

826 understanding or accuracy on the coach's part. Empathic understanding and accuracy are critical

827 components of co-orientation, and the use of these two facets may provide the coach with cues or

828 signals about an athlete's need for social support. When empathic understanding and accuracy

829 are present, there often increases in successful and effective coach-athlete relationships (Lorimer

830 \& Jowett, 2013).

831 Injured athletes' need for social support has been well documented, and it has been

832 shown that the support is typically not coming from the coaches even when the athletes desire it

833 (Corbillon et al., 2008; Gould et al., 1997; Udry et al., 1997). Successful coach-athlete

834 relationships should demonstrate congruence in co-orientation between coaches and athletes,

835 which would include the coach's ability to understand an athlete's need for support. 
APPENDIX B: ASSUMPTIONS, KEY TERMS, AND LIMITATIONS

\section{Assumptions}

838 1. It is assumed that the participants will be honest in their responses during interviews.

839 2. It is assumed that coaches can reflect on the injury experience.

\section{Definition of Key Terms}

841 1. Coach - Someone who is currently employed as the head coach at a National Collegiate

842 Athletic Association Division I university.

843 2. Injury - "when the evaluation identifies the complaint to be sufficient to require the player to 844 be restricted from participation" (Powell \& Dompier, 2004, p. 58).

845 3. International Sport Coaching Framework (ISCF) - A framework developed by the

846 International Council for Coaching Excellence (ICCE, 2014) to demonstrate the three types of

847 knowledge (i.e., professional, interpersonal, intrapersonal) needed to be an effective coach.

848 4. Rehabilitation - the process of recovering from an injury through means such as using ice or 849 heat, stretching, resistance training, and/or physical therapy, although this is not an exhaustive 850 list.

851 5. Social support - "an exchange of resources between at least two individuals perceived by the 852 provider or the recipient to be intended to enhance the well-being of the recipient" (Shumaker \& 853 Brownell, 1984, p. 13)

854 a. Emotional support refers to providing a sense of comfort and care to the recipient (e.g. 855 injured athlete) or being the shoulder to cry on.

856 b. Informational support refers to giving advice about the athlete's situation or providing 857 them with information about their injury and rehabilitation process. 
c. Tangible support can be described as providing concrete assistance to the injured athlete (e.g. giving the athlete a ride to a doctor's appointment).

$860 \quad$ Limitations

861 1. Being a qualitative study, the results will be limited to the perspectives of the participants from 862 a handful of Division I athletic organizations across the country.

863 2. Socially desirable responses are a limitation of any study that examines human behavior. 


\section{APPENDIX C: RESEARCHER POSITIONALITY}

As a former athlete, I have my own experiences with injury that spanned across my years of participation. I have had injuries that required rest and ice and those where I broke bones and had to miss practice and games. These experiences and those of my teammates are what inspired me to research the psychology of injury. As I reflect on my sport participation through the lens of a sport psychology researcher, I can also acknowledge that my injuries may have been perceived differently than my male counterparts because of my gender. Hypermasculinity within sport culture impacts how athletes navigate their injury experience as they live within the sport ethic. Sport is a male pursuit in society; male athletes are mocked or criticized for showing a response to pain and injury. As a female athlete, I was under less pressure than my male counterparts to adhere to the sport ethic. Ascribing to the sport ethic means that athletes play through their pain and reporting any injuries or pain is a sign of weakness. The sport ethic demands that we make sacrifices for our sport, which may often include our physical health. During my time as an athlete, I often tried to hide my own injuries because I did not want to miss practice or games or jeopardize my spot in the line-up, like many other injured athletes. However, my experience is limited to that of a female, and I could not claim to understand the impact that a hypermasculine sport culture has on a male who sustained an injury. As I spoke with male coaches about their male athletes, this was a critical piece to clarify.

I was both an insider and an outsider to my participants, and there was no one specific way to define what this means for my sample because my status as a white, heterosexual, cisgender female, former basketball player, and current sport psychology researcher will be perceived differently by each participant. As a former athlete, I have knowledge about the injury experience from the athlete's perspective through my participation and the literature. I am 
887 seeking to highlight the coach's experience with injured athletes because researchers know so 888 little about how coaches navigate the injury recovery process. To coaches, I am likely to be 889 perceived as more of an outsider because I have no experience as a coach but my father is a high 890 school coach, and my knowledge of the ins and outs of coaching is limited to what I have seen 891 through his experiences. In the interviews, I shared the information about my perspective on 892 coaching and my desire to give coaches a chance to share what the injury rehabilitation 893 experience is like for them. Coaches responded very positively to my both my perspective and 894 experiences with coaching, as well as my desire to include them in the research surrounding their 895 coaching behaviors.

Building rapport was essential to the success of the interview process. As an outsider to

897 the context of college athletics and the coaches' environments, I had to engage with the coaches 898 in a way that would allow coaches to see my intentions behind the interview. Some of the 899 coaches were hesitant to divulge too much information, perhaps because they were unsure of my 900 intentions or fear of negative repercussions. Most of the coaches, however, were excited to hear 901 that their voices were what I valued in this interaction and how it would inform my 902 understanding of what it is like to coach injured athletes.

903 As I collected data from participants, it was important to me as a researcher to stay true to 904 my epistemology, social constructivism. Social constructivism, as derived from constructionism, 905 suggests that the generation of meaning is always social and that culture is responsible for our 906 thoughts and how we come to understand the world. I aligned myself with social constructivism 907 to explore the perspectives of the coaches involved to create a more extensive understanding of 908 the dynamics of the coach-athlete relationship during injury. Social constructivism focuses on 909 how individuals make meaning of their interactions with the world around them (Crotty, 1998). 
910 That is, rather than accepting there is an absolute truth to the world, social constructivists take

911 the view that our understanding of the world and our interactions within it is constructed based

912 on "knowledge and reality constructed through discourse or conversation" (Sommers-Flanagan

913 \& Sommers-Flanagan, 2015, p. 370). Social constructivists focus on the discourse between

914 individuals as they come together to create their reality.

915 Culture is a relevant influence on how my participants come to understand their world,

916 but my role was not to criticize the culture the participant exists within, but to explore their

917 understanding of their world and experiences (Crotty, 1998). The theoretical lens I used to

918 address my research questions is that of an interpretivist as I look for "culturally derived and

919 historically situated interpretations of the social life-world" (Crotty, 1998, p. 67). Culture, and

920 our understanding of culture, is constructed from our experiences. Our inquiry as social

921 constructivists and interpretivists focuses on the "process by which meaning is created,

922 negotiated, sustained, and modified within a specific context of human action" (Schwandt, 1998,

923 p. 225). Coaches approached their injured athletes based on their own experiences with injury

924 and the interactions they have had with the athletes on their team.

925 With this being my first qualitative study, I spent a lot of time reflecting on how I came

926 to understand my epistemology and if that came first or if my research questions influenced my

927 epistemology. My epistemology was informed by the research questions, yet I had not been as

928 informed about my stance on knowing and understanding I developed these questions. From the

929 interpretivist lens used, I framed inquiry into the behaviors of coaches as an interpretation of

930 their world, where I focused on finding meaning over objectivity. Taking a social constructivist

931 approach to this research topic has created a more vivid depiction of what this experience looked

932 and felt like for my participants. Had I taken more of a positivist stance on this topic, I would not 
933 have left room for the interpretations of my participants and in my opinion, missed valuable

934 information that illustrated their experiences. The world is experienced very differently for each

935 person in the world, and there cannot be one absolute truth as we all see the world through our

936 own unique lens and the coaches in this study were no exception. 
APPENDIX D: DATA COLLECTION

Semi-Structured Interview Guide

939 1. Describe how you develop relationships with your injured athletes.

940 2. Describe how you typically respond when your athletes first become injured.

941 3. How do you communicate or engage with your injured athletes while they are in 942 rehabilitation?

a. What do you perceive your role to be during this stage?

944 4. How do you communicate and/or engage with your athletes as they come back from injury and are capable of engaging in practice and competition? with an injured athlete?

a. Is there anything you wish you could have done differently when working with your injured athletes?

5. Thinking back on your experience with injured athletes as a DI coach, do you feel like there are circumstances that led you to alter your approach to communicating or engaging

6. In your experience as a DI coach, have you experienced any obstacles or barriers while trying to help your injured athletes?

8. What advice would you offer to other DI coaches who want to offer support their injured athletes?

9. How do you see your personal experiences with injury as an athlete, either yourself or seeing a teammate go through it, influencing how you coach your injured athletes? 
APPENDIX E: TABLES

960 Table 1. Participant demographic information

\begin{tabular}{|c|c|c|c|c|c|c|}
\hline Coach & Age & Region & Team & $\begin{array}{l}\text { Years as DI } \\
\text { coach }\end{array}$ & $\begin{array}{l}\text { Total Years } \\
\text { Coaching }\end{array}$ & $\begin{array}{l}\text { Professional education } \\
\text { and training on athletic } \\
\text { injury? }\end{array}$ \\
\hline Allison & 29 & East North Central & M/W Swimming & 4 & 6 & $\mathrm{Y}$ \\
\hline Claire & 43 & West North Central & W Gymnastics & 12 & 21 & $\mathrm{Y}$ \\
\hline David & 45 & East South Central & W Soccer & 2 & 19 & $\mathrm{Y}$ \\
\hline Emily & 59 & West North Central & W Rowing & 23 & 26 & $\mathrm{~N}$ \\
\hline Howard & 52 & East South Central & Baseball & 3 & 3 & $\mathrm{Y}$ \\
\hline Joe & 52 & Mountain & W Golf & 9 & 17 & $\mathrm{~N}$ \\
\hline Kevin & 61 & Mountain & W Basketball & 17 & 29 & $\mathrm{Y}$ \\
\hline Lauren & 32 & Middle Atlantic & W Soccer & 2 & 4 & $\mathrm{Y}$ \\
\hline Mason & 62 & Pacific & Football & 20 & 31 & $\mathrm{Y}$ \\
\hline Noah & 47 & Mountain & M/W Tennis & 5 & 20 & $\mathrm{~N}$ \\
\hline Owen & 42 & West South Central & M Basketball & 1 & 17 & $\mathrm{Y}$ \\
\hline Tanya & 30 & South Atlantic & W Volleyball & 1 & 8 & $\mathrm{Y}$ \\
\hline Wyatt & 42 & New England & W Ice Hockey & 6 & 15 & $\mathrm{~N}$ \\
\hline
\end{tabular}


962 Table 2. A visual representation of how the interview guide addresses the three knowledge types

963 of the ISCF.

Professional Interpersonal Intrapersonal

\begin{tabular}{lll}
\hline $\begin{array}{l}\text { Describe your process of how you develop } \\
\text { relationships with your injured athletes. }\end{array}$ & $\mathrm{x}$ & $\mathrm{x}$ \\
\hline $\begin{array}{l}\text { In what ways do you communicate and/or engage } \\
\text { with injured athletes when they first become } \\
\text { injured? }\end{array}$ & $\mathrm{x}$ & \\
\hline
\end{tabular}

How does your communication or level of engagement vary, if at all, while the athlete is engaged in their rehabilitation? What do you

$\mathrm{X}-\mathrm{X}$

perceive your role to be during this stage?

How do you communicate and/or engage with your athletes as they come back from injury and are capable of engaging in practice and competition?

Thinking back on your experience with injured athletes as a DI coach, do you feel like there are circumstances that led you to alter your approach

X $\quad \mathrm{X}$

to communicating or engaging with an injured athlete?

Is there anything you wish you could have done differently when working with your injured athletes?

In your experience as a DI coach, have you experienced any obstacles or barriers while trying to help your injured athletes?

Have you ever reached out to others or sought out additional information to educate yourself or your athlete more about injury?

What advice would you offer to other DI coaches who may be struggling to support their injured athletes?

How do you see your personal experiences with injury as an athlete, either yourself or seeing a teammate go through it, influencing how you

$\mathrm{X}$

$\mathrm{X}$ coach your injured athletes? 
965 Table 3. Code map of iterations of analysis for professional knowledge (to be read from bottom up; Anfara et al., 2002)

Professional Knowledge

Theme 1: Professional Knowledge on Resources
Theme 2: Professional Knowledge of Injury Prevention
Theme 3: Professional Knowledge on Phases of Injury Rehabilitation

\begin{tabular}{lllll}
\hline & & Second iteration: Sub-themes & \\
1a Network roles & $2 \mathrm{a}$ & Injury Prevention & $3 \mathrm{a}$ & Managing emotional responses \\
$1 \mathrm{~b}$ & Accessibility of resources & & $3 \mathrm{~b}$ & Performance decrements \\
\hline
\end{tabular}

First iteration: Categories developed from initial codes

1a Listen to the medical staff

1a Use depends on role

1a Sport psychology

1a Trust expertise

1b Access to great doctors

1b Information readily available

1b Larger schools have more resources

1b Access to facilities for lower-tier sports

1b Finances and insurance 2a Proper training techniques to reduce injury

2a Stay up to date

2a Use your resources

2a Mental aspects of recovery 3a Visualize different scenarios

3a Remain calm

3a Frustration

3b Lack of conditioning

$3 b \quad$ Poor performance

$3 \mathrm{~b}$ Severity impacts involvement 
967 Table 3 (cont.). Code map of iterations of analysis for professional knowledge (to be read from bottom up; Anfara et al., 2002)

Theme 4: Professional Knowledge on Injured Athletes' Sustained Theme 5: Professional Knowledge on Athlete Welfare Involvement

Second iteration: Sub-themes

4a Injured athletes' sustained involvement

5a Decisions on return to play

$5 b \quad$ Person-first focus

First iteration: Categories developed from initial codes

4a Create unique workouts

4a Support and encourage teammates

4a Assist with coaching tasks 5a Know your players

5 a Listen to the athlete

5a Listen to the medical staff

5a Do not overstep your boundaries

5 b Know your athletes' limits

5 b Do not add more stress about their return to play

$5 b \quad$ Focus on your player, not your win loss record 
974 Table 4. Code map of iterations of analysis for interpersonal knowledge (to be read from bottom up; Anfara et al., 2002)

Interpersonal Knowledge

Theme 1: Interpersonal Knowledge on Communicating with Injured Athletes
Theme 2: Interpersonal Knowledge on Perceiving Athletes as Barriers

\section{Second iteration: Sub-themes}

1a Communication as a support tool

1b Challenges in communication 2a Athletes' unrealistic expectations

$2 b$ Athletes' dishonesty

First iteration: Categories developed from initial codes

1a Do not talk at them

1a Talk face to face when possible

1a Pay attention to body language

1a Maintain contact

1a Ask how they feel

$1 b \quad$ Set clear expectations

1b Dividing attention between injured athletes and the team

1b Ask for feedback 2a Coach focuses on healthy athletes

2a Athletic trainers are a quick fix for injuries

$2 b$ Unsure if athletes are truthful

$2 \mathrm{~b}$ Fear of being benched

$2 b$ Concern about under reporting 
980 Table 5. Code map of iterations of analysis for intrapersonal knowledge (to be read from bottom up; Anfara et al., 2002)

Intrapersonal Knowledge

Theme 1: Intrapersonal Knowledge on Personal Coaching Experiences
Theme 2: Intrapersonal Knowledge on Personal Athletic Experiences

Second iteration: Sub-themes

1a Personal coaching experiences

2a Personal athletic experiences

First iteration: Categories developed from initial codes

1a Get better with experience

1a Ask more questions

1a Take individualized approach

1a Treat everyone equally

1a Reflect on what injury means to the athlete

1a Awareness of athletes struggles 2a History of injury experiences

2a What do I wish was done differently?

2a More medical advancements now 


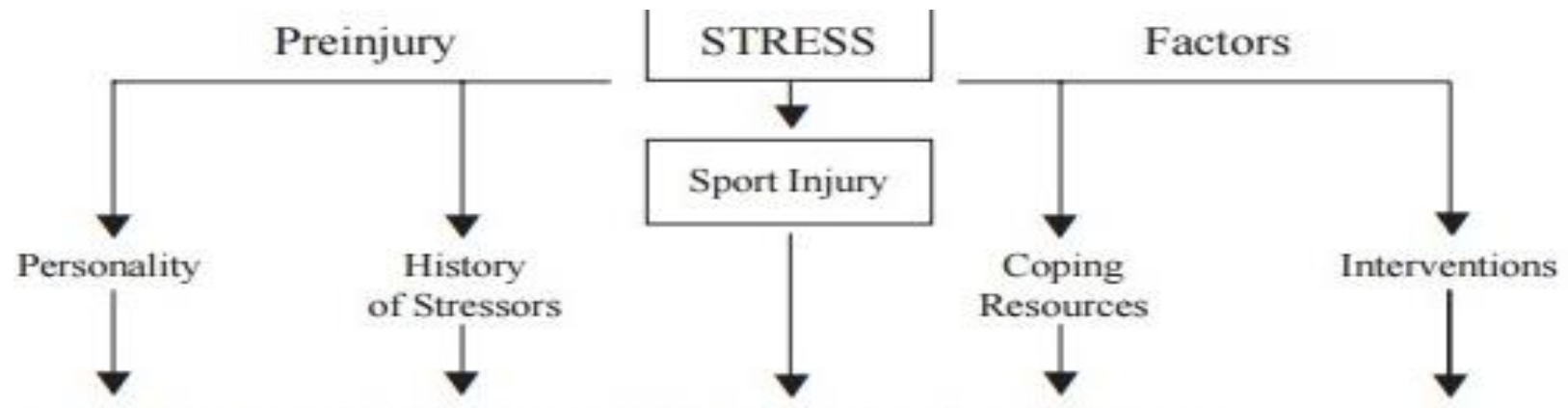

\section{RESPONSE TO SPORT INJURY AND REHABILITATION PROCESS}

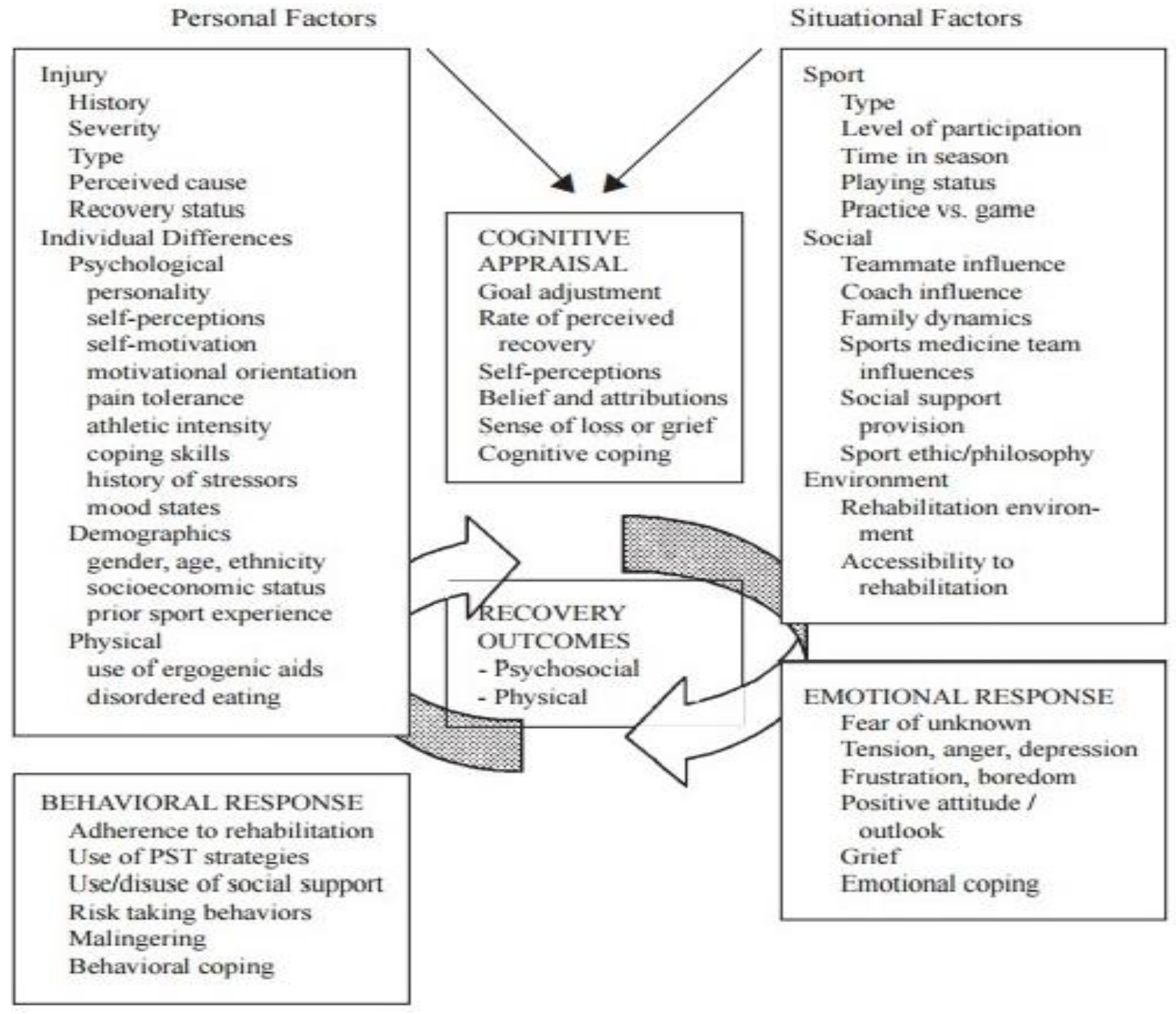

